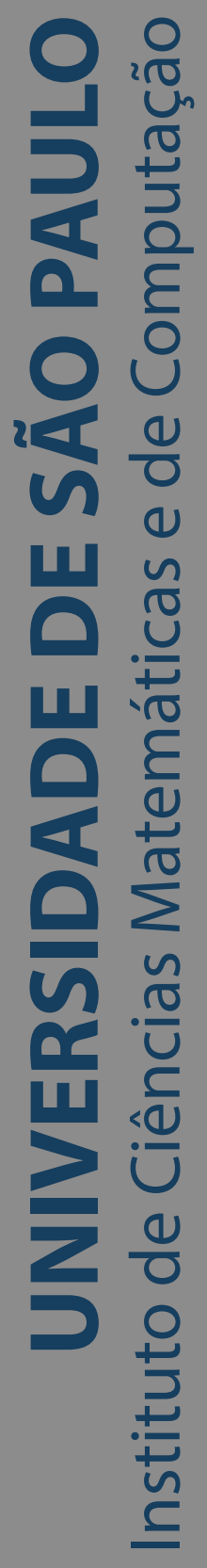

\title{
Propagação de epidemias em redes adaptativas
}

\section{Bruno Daniel Sanches da Silva}

Dissertação de Mestrado do Programa de Pós-Graduação em Ciências de Computação e Matemática Computacional (PPG-CCMC) 

Data de Depósito:

Assinatura:

\section{Bruno Daniel Sanches da Silva}

\section{Propagação de epidemias em redes adaptativas}

Dissertação apresentada ao Instituto de Ciências Matemáticas e de Computação - ICMC-USP, como parte dos requisitos para obtenção do título de Mestre em Ciências - Ciências de Computação

e Matemática Computacional. EXEMPLAR DE DEFESA

Área de Concentração: Ciências de Computação e Matemática Computacional

Orientador:: Prof. Dr. Francisco Aparecido Rodrigues

\section{USP - São Carlos}

de 2019 
Ficha catalográfica elaborada pela Biblioteca Prof. Achille Bassi e Seção Técnica de Informática, ICMC/USP, com os dados inseridos pelo(a) autor(a)

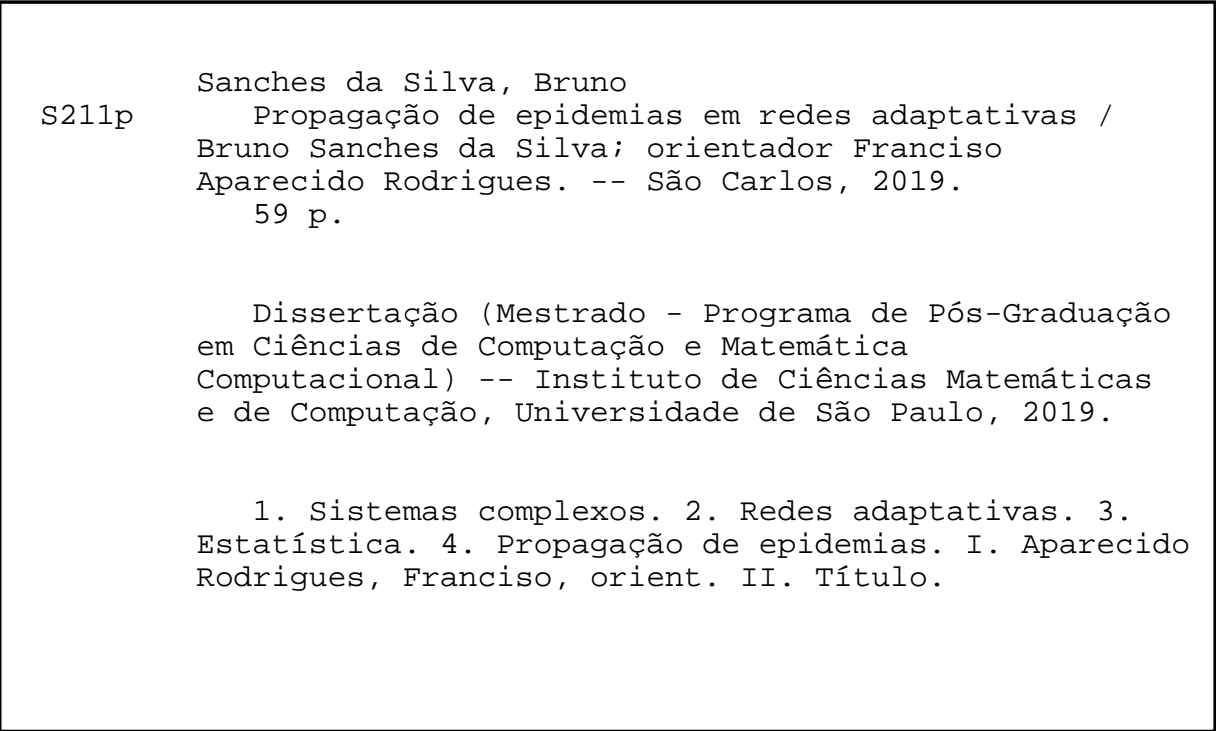

Bibliotecários responsáveis pela estrutura de catalogação da publicação de acordo com a AACR2: Gláucia Maria Saia Cristianini - CRB - 8/4938 Juliana de Souza Moraes - CRB - 8/6176 


\section{Bruno Daniel Sanches da Silva}

\section{Epidemic spreading in adaptive networks}

Master dissertation submitted to the Institute of Mathematics and Computer Sciences - ICMC-USP, in partial fulfillment of the requirements for the degree of the Master Program in Computer Science and Computational Mathematics. EXAMINATION BOARD PRESENTATION COPY

Concentration Area: Computer Science and Computational Mathematics

Advisor: Prof. Dr. Francisco Aparecido Rodrigues

\section{USP - São Carlos}

2019 

Este trabalho é dedicado a todas as pessoas que se interessam por essa incrível área que é redes complexas. 

Os agradecimentos principais são direcionados a todos os professores, a minha família e a todas as pessoas que me deram suporte ao longo de todos esses anos de ICMC 

"Expect problems and eat them for breakfast." Alfred A. Montapert 



\section{RESUMO}

SILVA, B. D. S. Propagação de epidemias em redes adaptativas. 2019. 57 p. Dissertação (Mestrado em Ciências - Ciências de Computação e Matemática Computacional) - Instituto

de Ciências Matemáticas e de Computação, Universidade de São Paulo, São Carlos - SP, 2019.

Modelos de propagação de epidemias e rumores são fundamentais para a previsão e controle da transmissão de agentes infecciosos e comportamento social. A maioria dos modelos desenvolvidos em redes complexas considera apenas estruturas estáticas, onde a conexões são mantidas ao longo do tempo. Apenas recentemente, modelos de propagação de epidemias em redes adaptativas foram propostos. Nessas redes, as conexões não são estáticas, variando durante a propagação do agente infeccioso. Neste trabalho proporemos um modelo de movimentação de agentes e iremos analisar a dinâmica entre o comportamento individual dos agentes e a propagação de epidemias e rumores. Os resultados obtidos permitirão uma melhor compreensão sobre a propagação de informação em redes complexas com estrutura variante no tempo.

Palavras-chave: Sistemas Complexos, Redes Adaptativas, Estatística, Propagação de epidemias. 



\section{ABSTRACT}

SILVA, B. D. S. Epidemic spreading in adaptive networks. 2019. 57 p. Dissertação (Mestrado em Ciências - Ciências de Computação e Matemática Computacional) - Instituto de Ciências Matemáticas e de Computação, Universidade de São Paulo, São Carlos - SP, 2019.

Propagation models of epidemics and rumors are fundamental to forecast and control the transmission of infectious agents and social behavior. Most models developed in complex networks consider only static structures where connections are maintained over time. Only recently, epidemic models in adaptative networks have been proposed. In these networks, the connections are not static, varying during the spread of the infectious agent. In this study, we aimed to analize the dynamics that emerges from the interplay between the individual behavior of agents and the spreading of an epidemic or rumor. The results allow a better understanding of the spread of information in complex networks with time-varying structure.

Keywords: Complex Systems, Adaptative networks, Statistics, epidemic spreading. 



\section{LISTA DE ILUSTRAÇÕES}

Figura 1 - Resposta temporal dos modelos para propagação de epidemias. (a) Modelo SIR: mostra as frações da população susceptíveis, infectadas e recuperadas. Os parâmetros desta simulação foram $\beta=1, \gamma=0.2, s_{0}=0.99, x_{0}=0.01$ e $r_{0}=0$; (b) Modelo SIS: a fração dos indivíduos infectados neste modelo cresce de acordo com a curva logística, entretanto, a fração de infectados nunca chega a 1. Figuras adaptadas de (NEWMAN, 2010) . . . . . . . . . . 26

Figura 2 - Exemplo de propagação de epidemia no modelo SIS em uma rede ER para três taxas $\mu$ diferentes. Para esse exemplo a taxa de religação $\alpha=0 \quad \ldots .31$

Figura 3 - Quantidade de infectados conforme a taxa $\beta$ de infecção varia, para diversos valores de taxa de religação. É possível notar que a doença demora mais para conseguir sobreviver na população conforme aumenta-se o valor de $\alpha$. . . 32

Figura 4 - Comparação entre redes para um $\beta$ fixo. . . . . . . . . . . . 33

Figura 5 - A infecção começa a crescer até que uma grande parte da população começa a se recuperar, nesse momento a epidemia começa a diminuir. Para essa simulação foi utilizado $\beta=0.0050 \ldots \ldots$. . . . . . . . . . 34

Figura 6 - Evolução da doença ao longo do tempo para diferentes taxas $\beta$. É possível notar que quanto maior o $\beta$ mais rápido a epidemia cresce e consequentemente, mais rápido termina. . . . . . . . . . . . . . . . . . . 35

Figura 7 - Comparação entre a propagação da epidemia com e sem religação. . . . . . 36

Figura 8 - Modelo SIR executado na rede ER para diferentes taxas de religação. É possível notar que a taxa $\alpha$ tem pouca influencia sobre a rede, sendo necessária altos valores para que a rede demonstre alguma diferença visível no resultado. Para esses experimentos foi utilizado $\beta=0.007 \ldots \ldots$. . . . . . . .

Figura 9 - Comparação entre a taxa critica de infecção e o valor minimo para que os agentes se movam. Podemos notar que existe um ponto onde a taxa critica atinge seu máximo. Foram utilizados três valores diferentes da taxa de recuperação $\mu$. A velocidade utilizada foi de $V=0.3$. Raio de contato $r=0.6$. Desvio padrão médio para o calculo dos $\beta_{c}$ de $0.000130 \ldots$. . . . 
Figura 10 - (a) Número médio $<k>$ de vizinhos de um nó diminui com o tempo. (b) Distância média $<d>$ entre dois vértices vizinhos aumenta de acordo com a passagem do tempo $t$. Foram utilizados os seguintes parâmetros: Taxa de infecção $\beta=0.15$, taxa de recuperação $\mu=0.4$, raio de contato $r=0.45$, velocidade de movimento $v=0.3$ e limiar de movimento $E=7$. Gráficos na escala logarítmica. Desvio padrão para o calculo de (a) 0.000158. Desvio padrão para o calculo de (b) 0.000095 . . . . . . . . . . . . . . .

Figura 11 - (a) Número de componentes conexas ao longo do tempo. (b) Porcentagem de indivíduos presentes na maior componente conexa ao longo do tempo. Foram utilizados os seguintes parâmetros: Taxa de propagação do rumor $\beta=0.35$, taxa de recuperação $\mu=0.1$, raio de contato $r=0.45$, velocidade de movimento $v=0.3$, limiar $E=7$. Desvio padrão para o calculo de (a) 0.000158. Desvio padrão para o calculo de (b) 0.000348 . . . . . . . . . . .

Figura 12 - (a) Número de agentes versus porcentagem de infectados. É possível notar que a porcentagem de infectados cresce exponencialmente com o aumento do número de agentes (b) Raio de contato versus porcentagem de infectados. $\mathrm{O}$ mesmo efeito que ocorreu para a densidade de agentes também ocorre para a variação do raio de contato. Foram utilizados os seguintes parâmetros: Taxa de propagação do rumor $\beta=0.35$ (Quando não especificado), taxa de recuperação $\mu=0.1$, raio de contato $r=0.45$, velocidade de movimento $v=0.3, E=2$ (Quando não especificado). Desvio Padrão de (a) 0.000474 . Desvio padrão de (b) $0.000032 \ldots$. . . . . . . . . . . . . . .

Figura 13 - Velocidade dos agentes versus \% de infectados. O Raio de contato utilizado foi $r=0.45$, Taxa de propagação do rumor $\beta=0.35$, taxa de recuperação $\mu=0.3$ Desvio padrão de $0.000221 \ldots \ldots$. . . . . . . . . .

Figura 14 - Comparação da taxa $E_{\text {opt }}$ para diferentes valores de $H$. A taxa de recuperação $\mu$ utilizada foi de 0.3 . A velocidade utilizada foi de $V=0.3$. Raio de contato $r=0.6$. Desvio padrão médio (Soma dos desvios padrões para cada $\mathrm{H}$ ) $0.000063 \ldots \ldots \ldots \ldots \ldots \ldots \ldots \ldots$

Figura 15 - Comparação da porcentagem de infectados ao final do processo para diferentes valores de $H$. A taxa de recuperação $\mu$ utilizada foi de 0.3 . A velocidade utilizada foi de $V=0.3$. Raio de contato $r=0.6$ Desvio padrão 0.000158 .

Figura 16 - A taxa de recuperação $\mu$ utilizada foi de 0.3 . A velocidade utilizada foi de $V=0.3$. Raio de contato $r=0.6$ Desvio padrão $0.000370 \ldots \ldots$. . . . . 50

Figura 17 - A taxa de recuperação $\mu$ utilizada foi de 0.3 . A velocidade utilizada foi de $V=0.3$. Raio de contato $r=0.6$ Desvio padrão $0.000370 \ldots \ldots$. . . . . 
INTRODUÇÃO $\ldots \ldots \ldots \ldots \ldots \ldots \ldots$

REDES COMPLEXAS E MODELOS EPIDEMIOLÓGICOS . . . . 23

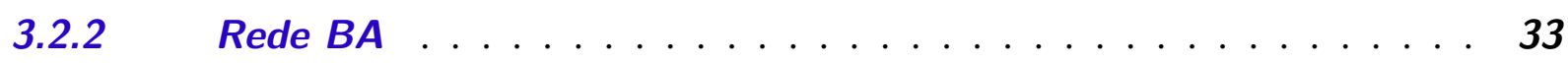

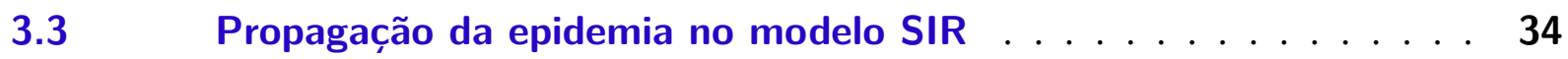

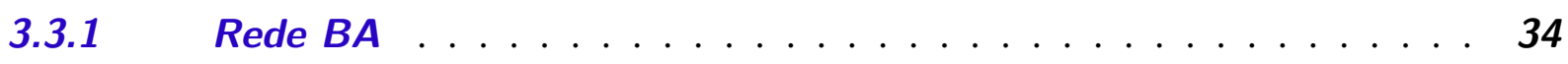

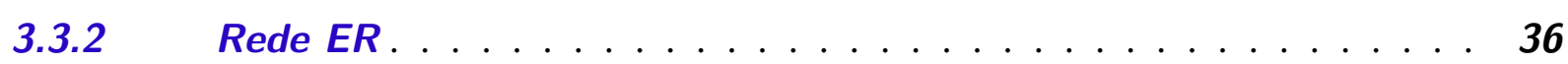

4 MODELOS EPIDÊMICOS COM MOVIMENTAÇÃO DE AGENTES 39

$4.1 \quad$ Propagação da epidemia no modelo SIS $\ldots \ldots \ldots \ldots$

5 EPIDEMIAS EM MEIOS HETEROGÊNEOS . . . . . . . . . 47

$5.1 \quad$ Fractal Landscape . . . . . . . . . . . . . . . . . . 47

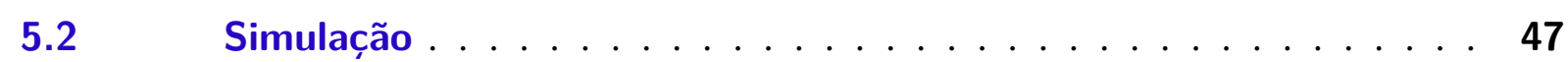

5.2.1 Propagação de epidemia no modelo SIS . . . . . . . . . . . 48

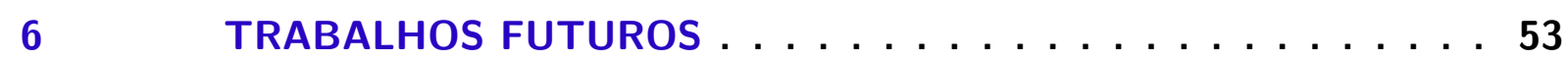

$6.1 \quad$ Conclusão e trabalhos Futuros $\ldots \ldots \ldots \ldots \ldots$ 
REFERÊNCIAS . . . . . . . . . . . . . . . . . 55 


\section{1}

INTRODUÇÃO

O estudo de redes complexas vem crescendo cada vez mais devido à sua grande generalidade na representação dos mais diversos sistemas complexos (BOCCALETTI et al., 2006; COSTA et al., 2011). Diversos estudos têm mostrado que a estrutura da rede influencia de forma fundamental a maioria dos processos dinâmicos (BOCCALETTI et al., 2006; BARRAT; BARTHELEMY; VESPIGNANI, 2008). Com isso, grande parte dos esforços da comunidade científica se concentra em determinar como propriedades topológicas de redes afetam a evolução da propagação de epidemias (PASTOR-SATORRAS et al., 2015),a sincronização de osciladores acoplados (RODRIGUES et al., 2015), ou a robustez de redes complexas (ALBERT; JEONG; BARABÁSI, 2000). Esse estudo permite não apenas entender a relação entre topologia e dinâmica de redes, mas também desenvolver métodos para prever e controlar a evolução de processos dinâmicos. A propagação de epidemias e rumores é um dos processos dinâmicos cuja evolução é altamente influenciada pela organização de rede (PASTOR-SATORRAS et al., 2015). Por exemplo,a taxa de propagação mínima para ocorrência de um estado epidêmico depende inversamente do segundo momento estatístico da distribuição do número de conexões (PASTOR-SATORRAS; VESPIGNANI, 2001b). Desse modo, em redes sem escala, tal coeficiente é nulo, o que torna essas redes meios ideais para propagação de epidemias (PASTOR-SATORRAS; VESPIGNANI, 2001b). Várias outras propriedades da rede, tais como assortatividade e organização modular, também afetam a propagação de epidemias (PASTOR-SATORRAS et al., 2015).

Apesar dos avanços no estudo da relação entre a estrutura da rede e propagação de epidemias e rumores, a maioria dos trabalhos utiliza redes cuja estrutura se mantém estática durante a propagação do agente infeccioso (PASTOR-SATORRAS et al., 2015). No entanto, em casos reais, a rede se organiza durante a manifestação de tal agente, de modo que indivíduos suscetíveis diminuam as suas chances de infecção (GROSS; BLASIUS, 2008). O mesmo ocorre na propagação de rumores, uma vez que as conexões entre pares de indivíduos não é estática, mas variável temporalmente. 
Neste trabalho, inicialmente será feita uma analise de como diferentes mecanismos de adaptação alteram a propagação de epidemias. Será proposto então, uma versão implícita de redes que mudam sua topologia com o tempo. Agentes são distribuídos em um plano com condições de contorno periódicas, representando os nós da rede. Esses agentes podem mover-se baseando-se apenas em informações locais. Dois agentes são conectados se a distancia entre eles for menor que um limiar especificado. Essa dinâmica entre os nós causa uma constante mudança na topologia. Por fim será feita uma analise de como esses agentes comportam-se quando o meio em que se movem torna-se heterogêneo

\section{$1.1 \quad$ Hipóteses}

A principal hipótese desse trabalho é que é possível construir modelos de redes dinâmicas e modelar a propagação de epidemias e rumores nessas redes, obtendo resultados mais gerais do que os que consideram redes estáticas. Uma outra hipótese é que meios heterogêneos afetam a propagação de epidemias e rumores.

\subsection{Objetivo}

O objetivo desse trabalho é implementar e analisar modelos já existentes na literatura de redes complexas com interações dinâmicas, que mudam ao longo do tempo. Após analise dos modelos do estado da arte, será proposto um novo modelo de movimentação de agentes. Um processo epidêmico sera executado nessa rede e sera feita uma analise sobre os efeitos que surgem da dinâmica entre a movimentação e a propagação da epidemia.

Na parte final do trabalho, será demonstrado a relação que o meio tem sobre a propagação da epidemia. Relevos serão gerados e uma analise será feita sobre como esses relevos alteram a forma como a epidemia se propaga.

\subsection{Atividades}

A principal finalidade deste trabalho é analisar a dinâmica entre o comportamento individual dos agentes e a propagação de epidemias e rumores. Para isso serão discutidas implementações dos principais modelos de propagação. Mais especificamente serão realizados nesse trabalho:

- Redes Complexas: Será realizada uma breve análise dos principais artigos sobre redes complexas para ambientar o problema. Dentre os trabalhos principais, será considerado o trabalho de (BARABÁSI, 2016). 
- Propagação de epidemias e rumores: Será realizado um estudo sobre os principais modelos de propagação de epidemias e rumores. Para isso, será utilizado o livro de (PASTORSATORRAS et al., 2015).

- Redes adaptativas: Os principais trabalhos dessa área serão revisados incluindo (GROSS; BLASIUS, 2008). Serão implementados os modelos discutidos em (GROSS; D’LIMA; BLASIUS, 2006) e analisaremos seus resultados.

- Movimentação de agentes: Um novo modelo de movimentação de agentes será proposto e seus resultados discutidos. Além disso o modelo também será analisado em ambientes heterogêneos.

\subsection{Organização}

No capítulo 2 serão discutidos os principais trabalhos em redes complexas e algumas definições importantes.

No capítulo 3, será feita uma analise de alguns trabalhos já realizados na área de redes complexas adaptativas e serão feitos experimentos para demonstrar algumas propriedades desse tipo de rede.

No capítulo 4, será proposto uma versão implícita de redes adaptativas onde a movimentação de agentes causa uma constante mudança na topologia. Veremos quais as propriedades que emergem da interação entre agentes e a propagação de uma epidemia.

Por fim, no capítulo 5, analisaremos qual a influencia que o meio onde os agentes se movimentam tem sobre o modelo. 



\section{REDES COMPLEXAS E MODELOS EPIDEMIOLÓGICOS}

\subsection{Redes complexas}

Redes complexas existem em diversas aplicações do mundo real, seja na biologia (como em estudos sobre o clima) (YAMASAKI; GOZOLCHIANI; HAVLIN, 2008), seja em redes de computadores (BARABÁSI, 2016) ou em qualquer outra área do conhecimento, redes complexas fornecem uma ferramenta poderosa para modelar diversos tipos de problemas.

Uma rede complexa é um grafo que possuí propriedades topológicas não triviais que comumente acontecem em grafos que modelam sistemas reais. Tais propriedades incluem, distribuição de grau seguindo uma distribuição do tipo lei de potência, um coeficiente de aglomeração alto, assortatividade ou disassortatividade entre os vértices, entre outras (BOCCALETTI et al., 2006; BARABÁSI, 2016). Uma rede complexa que muda sua topologia ao longo do tempo é denominada rede complexa adaptativa.

\subsubsection{Modelo Erdős-Rényi}

O modelo Erdôs-Rényi (ER) é um modelo simples de rede aleatória. Para cada aresta possível do grafo, é associada uma probabilidade $p$ de existência que independe de outras arestas (ERDOS; RÉNYI, 1960). Nesse tipo de rede, o número de arcos que se conectam a um determinado nó, também conhecido como grau do vértice, segue uma distribuição de Poisson.

\subsubsection{Modelo Barabási-Albert}

O modelo Barabási-Albert (BA) é um exemplo de rede sem escala. Uma rede sem escala é uma rede cuja distribuição do grau de seus vértices segue uma lei de potência (BARABÁSI, 2016). Um mecanismo que gera esse tipo de rede, é a preferência que novos nós tem de se 
conectar com outros que possuem grau elevado (BARABÁSI, 2016). Essa tendência faz com que poucos vértices fiquem altamente conectados, enquanto muitos fiquem com poucas conexões. Os nós de grau elevado são denominados $h u b$.

Conforme apresentado em (BARABÁSI, 2016), essas redes têm sido observadas em diferentes domínios de aplicação tais como a Internet, em redes de metabolismo e em redes de citações de artigos científicos.

\subsubsection{Rede Espacial}

Redes espaciais são formadas por vértices que estão localizados em um espaço com uma métrica definida (BARTHÉLEMY, 2011). Na maioria das aplicações praticas, o espaço é um plano cartesiano de duas dimensões e a métrica é a distância euclidiana. Nessas aplicações, arestas são criadas se a distância entre dois vértices é menor que um limiar especificado.

\subsection{Modelos epidemiológicos}

Modelos epidemiológicos são ferramentas utilizadas para estudar os mecanismos pelos quais doenças se espalham, auxiliando na prevenção de surtos ou no controle de epidemias (BRAUER, 2008). Os modelos tradicionais se baseiam em dois fatores principais:

- Compartimentização: Cada indivíduo é classificado de acordo com o estágio, ou classe, em que se encontra durante a propagação da doença. Os trabalhos mais tradicionais assumem que cada indivíduo pode estar em um dos três compartimentos: Recuperado, Suscetível e Infectado.

- Mistura Homogênea: Um indivíduo infectado infecta outros com a mesma probabilidade. Essa condição elimina a necessidade do conhecimento da rede de contatos especifica de cada elemento da população.

\subsubsection{Modelos SIS e SIR}

O modelo Suscetível-Infectado-Suscetível divide a população em dois compartimentos: Suscetível e Infectado. Um indivíduo infectado é aquele que contraiu a doença, enquanto um indivíduo suscetível por sua vez, é aquele que pode ser infectado. Uma vez infectado, um elemento da população pode voltar a tornar-se suscetível com uma certa taxa. A dinâmica deste modelo pode ser melhor representada como em reações químicas como mostra a Equação 2.1

$$
\begin{aligned}
S+I & \stackrel{\beta}{\rightarrow} 2 I, \\
I & \stackrel{\mu}{\rightarrow} S,
\end{aligned}
$$


onde + representa um contato entre pares de indivíduos e os parâmetros $\beta$ e $\mu$ representam as taxas (ou probabilidades, nos modelos discretos) de infecção e recuperação, respectivamente. Ou seja, a infecção é transmitida através de contatos entre indivíduos, sendo um infectado e outro suscetível, mas a recuperação ocorre como um processo espontâneo.

Em muitos casos, os indivíduos após infectados desenvolvem imunidade contra a doença e portanto não podem tornar-se suscetíveis novamente, nem infectar outros indivíduos. Tal modelo é denominado Suscetível-Infectado-Recuperado. Similar ao SIS, podemos representar as transições desse modelo como em reações químicas,

$$
\begin{array}{rll}
S+I & \stackrel{\beta}{\rightarrow} & 2 I, \\
I & \stackrel{\mu}{\rightarrow} & R .
\end{array}
$$

No entanto, um indivíduo adquire imunização (com taxa $\mu$ ) e torna-se recuperado.

No caso de redes totalmente conectada, as equações determinísticas que regem a propagação de epidemias podem ser obtidas considerando-se a lei de ação das massas (PASTORSATORRAS et al., 2015). Seja $\rho_{I}$ e $\rho_{S}$ a fração média de vértices infectados e suscetíveis, respectivamente. Assim, para os modelos SIS e SIR (PASTOR-SATORRAS et al., 2015),

$$
\begin{array}{r}
\frac{d \rho_{I}}{d t}=\beta \rho_{I} \rho_{S}-\mu \rho_{I} \\
\frac{d \rho_{S}}{d t}=-\beta \rho_{I} \rho_{S}+\theta \rho_{I}
\end{array}
$$

onde $\beta$ é a taxa de propagação da epidemia e $\mu$ é a taxa de recuperação de indivíduos infectados. Para o modelo SIS, temos $\theta=0$, enquanto que para o SIR, $\theta=1$. Resolvendo essas equações, temos que o número de indivíduos infectados cresce exponencialmente se $\beta>\mu$. A Figura 1 mostra o comportamento da fração de infectados, recuperados ou suscetíveis ao longo do tempo. 


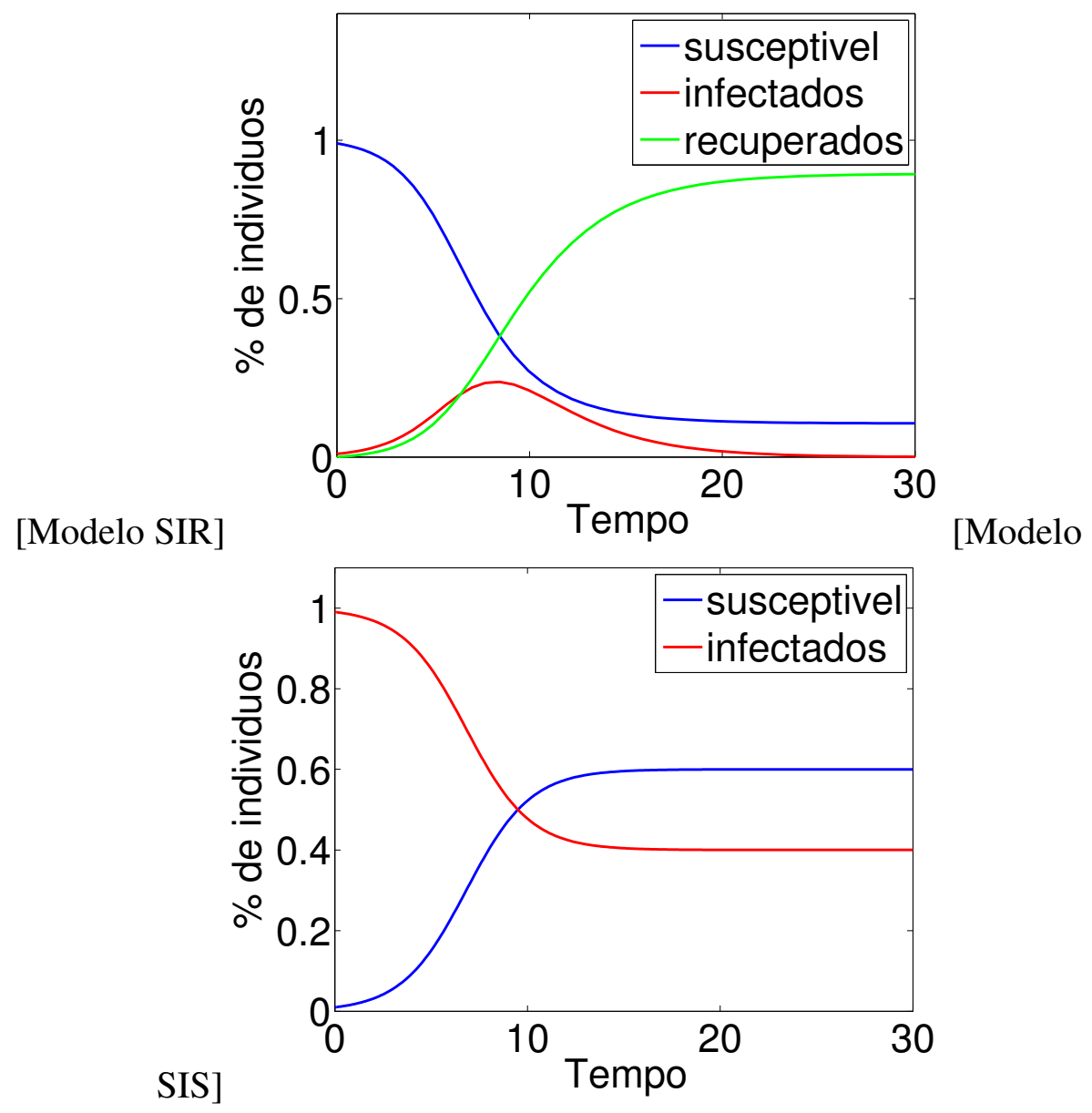

Figura 1 - Resposta temporal dos modelos para propagação de epidemias. (a) Modelo SIR: mostra as frações da população susceptíveis, infectadas e recuperadas. Os parâmetros desta simulação foram $\beta=1, \gamma=0.2, s_{0}=0.99, x_{0}=0.01$ e $r_{0}=0$; (b) Modelo SIS: a fração dos indivíduos infectados neste modelo cresce de acordo com a curva logística, entretanto, a fração de infectados nunca chega a 1. Figuras adaptadas de (NEWMAN, 2010)

\subsubsection{Propagação de rumores}

No caso da propagação de rumores, o interesse é propagar a informação da maneira mais eficiente possível, contrariamente ao que ocorre com as epidemias. O modelo padrão para propagação de rumor foi proposto há algumas décadas por Daley e Kendall (DALEY; KENDALL, 1965). Recentemente, muitos autores têm explorado este tópico com a aplicação de topologias de redes complexas (PASTOR-SATORRAS et al., 2015). O modelo apresenta três estados, denotados por I (ignorante), S (informantes, do inglês spreader) e R (contidos, do inglês stifler). Os ignorantes são aqueles indivíduos que não conhecem o rumor e são susceptíveis à informação. Os espalhadores são aqueles que já ouviram o rumor e estão espalhando o mesmo. Finalmente, contidos são aqueles que conhecem o rumor, mas não o espalham mais, ou seja, já perderam interesse por tal rumor.

O processo de propagação evolui pelo contato entre informantes e ignorantes. Quando um ignorante encontra um informante ele passa a ser um informante a uma taxa $\lambda$. O decai- 
mento deste ocorre por meio pelo esquecimento ou pela perda do interesse no rumor, assim os informantes tornam-se contidos com uma probabilidade $\alpha$ se estiverem em contato com outros informantes ou contidos. Podemos representa-lo como mostra a Equação 2.5

$$
\begin{aligned}
S+I & \stackrel{\beta}{\rightarrow} 2 I, \\
I+(I \vee R) & \stackrel{\mu}{\rightarrow} 2 R .
\end{aligned}
$$

Esta dinâmica é semelhante à propagação de epidemias SIR, porém, neste caso a recuperação é substituída pela perda de interesse no rumor e isto não ocorre de maneira espontânea, mas sim pelo contato entre informante e outros informantes ou informantes e indivíduos contidos. O desenvolvimento de teórico desse modelo pode ser visto em (NEKOVEE et al., 2007), onde é feita a análise do modelo derivando o limiar de propagação, considerando também um termo de esquecimento, no qual o indivíduo perde o interesse pelo rumor e passa de informante a contido espontaneamente. 



\section{3}

\section{MODELOS EPIDÊMICOS EM REDES ADAPTATIVAS}

Redes adaptativas são uma variação mais geral de redes comuns, uma vez que permitem modelar problemas mais complexos. Analisando-se uma das diversas aproximações para a solução analítica que podem ser utilizadas para descrever a dinâmica de propagação em redes estáticas (PASTOR-SATORRAS et al., 2015), podemos notar a importância que a topologia tem na propagação da epidemia. Essa aproximação é chamada aproximação de campo médio heterogênea (heterogeneous mean field). Nesse caso, assume-se que vértices com o mesmo número de conexões (mesmo grau) apresentam dinâmicas semelhantes. Sendo assim, seja $\rho_{k}^{I}(t)$ o número de nós infectados com grau $k$ no tempo $t, \lambda$ a taxa de transmissão, $\mu$ a taxa de recuperação e $\theta_{k}(t)$ a probabilidade média de que algum vizinho de qualquer vértice com grau $k$ esteja infectado, temos que o conjunto de equações que rege o sistema é dado por

$$
\frac{d \rho_{k}^{I}(t)}{d t}=-\rho_{k}^{I}(t)+\lambda k\left[1-\rho_{k}^{I}(t)\right] \theta_{k}(t),
$$

onde $\theta_{k}(t)=\sum_{k^{\prime}} P\left(k^{\prime} \mid k\right) \rho_{k^{\prime}}^{I}(t)$ e o tempo foi reescalado por $1 / \mu$ e $\lambda=\beta / \mu$. Resolvendo para redes não-correlacionadas (CATANZARO; BOGUNÁ; PASTOR-SATORRAS, 2005), temos que o número de infectados cresce se

$$
\lambda>\lambda_{c}^{\mathrm{DBMF}, \mathrm{unc}}=\frac{\langle k\rangle}{\left\langle k^{2}\right\rangle} .
$$

Ou seja, a taxa crítica de infecção depende do segundo momento da distribuição do número de conexões. Logo, a topologia da rede tem importância fundamental no processo de propagação.

A comunidade científica vem tentando analisar as propriedades que emergem quando são consideradas a dinâmica entre estado e topologia. Trabalhos recentes mostraram que alguns fenômenos aparecem repetidamente em redes adaptativas, tais como a observação de dinâmicas 
complexas (HOLME; NEWMAN, 2006; GROSS; D’LIMA; BLASIUS, 2006), auto-organização robusta (BORNHOLDT; ROHLF, 2000; HOLME; GHOSHAL, 2006) e o surgimento de diferentes classes em uma rede inicialmente homogênea (ITO; KANEKO, 2001).

\subsection{Simulações}

Foram simulados os modelos SIR e SIS em redes adaptativas enquanto um processo de epidemia se alastra pela rede. A simulação dos modelos foi feita utilizando o método de Monte Carlo em uma rede aleatória. Foi primeiramente fixada uma probabilidade $\beta$ de infecção, uma probabilidade $\mu$ de recuperação e uma probabilidade $\alpha$ de religação (ou rewiring). Foi também definida uma porcentagem da população como sendo os indivíduos inicialmente infectados e uma quantidade máxima de iterações que o método irá executar.

Para cada iteração, os indivíduos infectados primeiro tentam se recuperar com taxa $\mu$. Os nós que se recuperam não são excluídos do conjunto de indivíduos infectados, uma vez que isso só ocorrera no fim da iteração. Em seguida, cada indivíduo infectado tenta passar a doença para um vizinho $u$ que está saudável. Com probabilidade $p, u$ não percebe que seu vizinho está infectado e portanto pode ser contaminado. Com probabilidade complementar, $u$ percebe que seu vizinho possui a doença e tenta quebrar sua ligação com ele.

Em outras palavras, seja a variável $X_{u}$ o estado em que o vértice $u$ se encontra, a probabilidade de que o nó $u$ se torne infectado é $P\left(X_{u}=\right.$ Infectado $)=I_{u} * p * B$, onde $I_{u}$ é o número de vizinhos infectados que o nó $u$ possui. Já a probabilidade de que um par de vértices $u \mathrm{e}$ $v$ perca sua aresta, dado que $v$ está infectado é $P\left((\mathrm{u}, \mathrm{v})\right.$ perde aresta $\left.\mid X_{v}=\operatorname{Infectado}\right)=(1-p) * \alpha$

Uma vez que $u$ quebrou sua ligação, ele vai religar com outro nó escolhido de maneira aleatória que não esteja infectado.

\subsection{Propagação da epidemia no modelo SIS}

\subsubsection{Rede ER}

Em uma rede ER sem religação, com o aumento da probabilidade de infecção $\beta$, a rede tende a atingir um estado endêmico como mostrado na Figura 2 


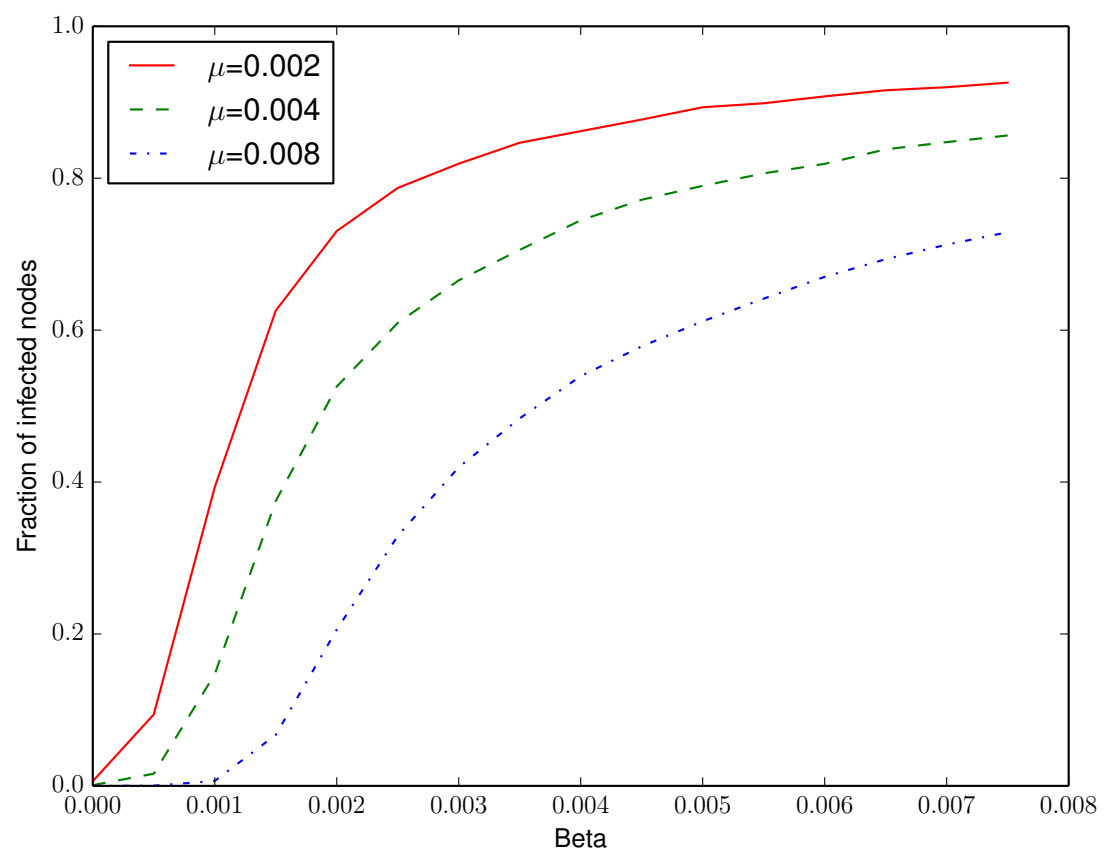

Figura 2 - Exemplo de propagação de epidemia no modelo SIS em uma rede ER para três taxas $\mu$ diferentes. Para esse exemplo a taxa de religação $\alpha=0$

A epidemia começa a crescer depois de um valor crítico denominado $\beta_{c}$. Para $\beta>\beta_{c}$, a epidemia sempre atinge o estado onde grande parte da população está infectada, denominado estado endêmico (PASTOR-SATORRAS; VESPIGNANI, 2001a). Na figura $2, \beta_{c}$ é muito próximo de zero e portanto esse limiar é quase imperceptível.

Como mostrado em GROSS; D'LIMA; BLASIUS o efeito que a religação tem no valor $\beta_{c}$ está ligado ao número básico de reprodução, denominado $R_{0}$, que representa a quantidade de indivíduos suscetíveis que são contaminados por um individuo infectado em uma população composta apenas por pessoas suscetíveis. Em uma rede que não possui religação, ou seja $\alpha=0$, o número básico de reprodução é $R_{0}=\frac{\beta\langle k\rangle}{\mu}$, onde $\langle k\rangle$ é o número médio de vizinhos de um vértice da rede. Se cada vértice do grafo contaminar pelo menos um outro vértice, a doença continuara a se espalhar e a epidemia nunca morrera. Portanto se assumirmos que $\beta=\beta_{c}$ então também devemos assumir que $R_{0}$ é pelo menos 1 . Substituindo esses dois termos na equação obtemos que a taxa $\beta_{c}=\frac{\mu}{\langle k\rangle}$.

Se a religação for levada em conta, um único nó infectado perde em média uma taxa $\alpha$ de suas arestas, logo seu grau ao longo do tempo pode ser definido como $k(t)=\langle k\rangle e^{-\alpha t}$ (GROSS; D'LIMA; BLASIUS, 2006). Assumindo $t$ como sendo o tempo médio em que um nó permanece infectado, ou seja, se $t=\frac{1}{\mu}$, temos que o valor $\beta_{c}$ pode ser definido como:

$$
\beta_{c}=\frac{\alpha}{\langle k\rangle\left[1-e^{\frac{-\alpha}{\mu}}\right]}
$$


Analisando a Equação 3.3 é possível perceber que quando $\alpha$ cresce, assumindo que $\mu$ permanece constante, $\beta_{c}$ também cresce. Em outras palavras, o limiar de infecção é diretamente proporcional a taxa de religação.

Esse efeito pode ser confirmado pelas simulações utilizando o algoritmo de Monte Carlo. A Figura 3 mostra como o valor $\beta_{c}$ varia de acordo $\operatorname{com} \alpha$

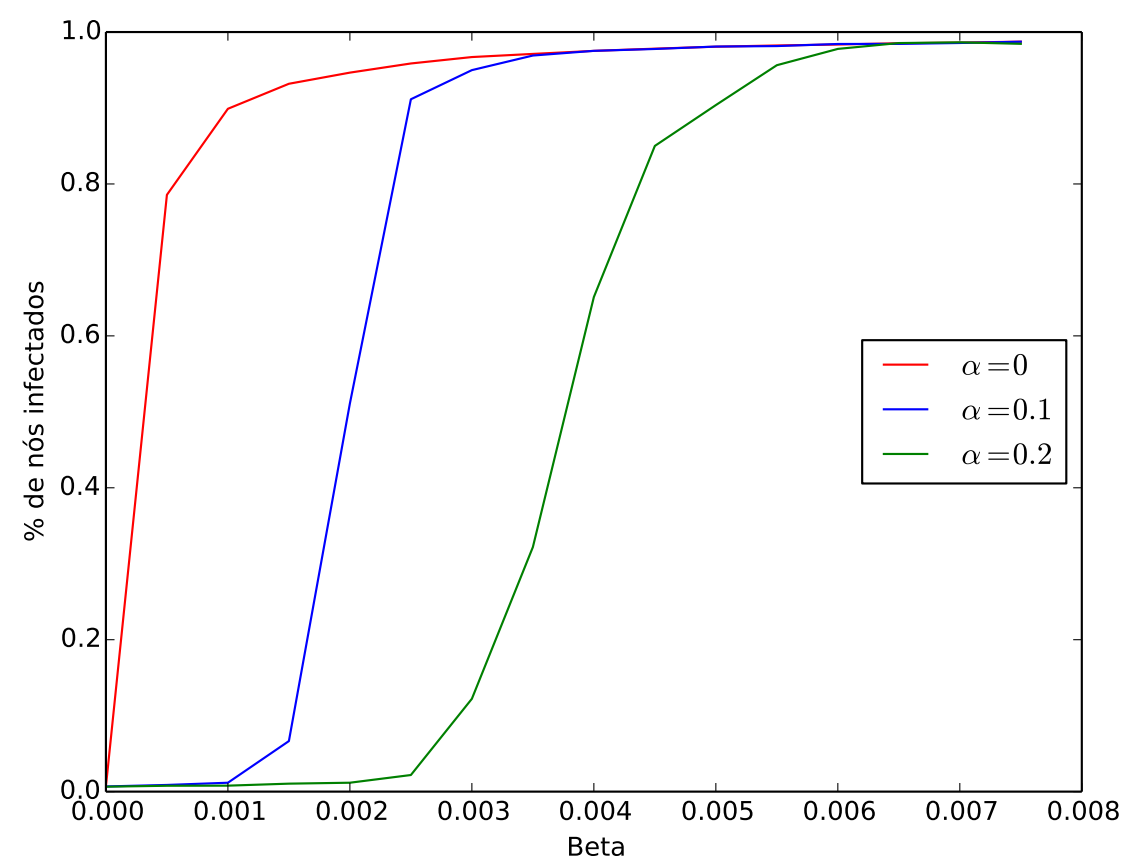

Figura 3 - Quantidade de infectados conforme a taxa $\beta$ de infecção varia, para diversos valores de taxa de religação. É possível notar que a doença demora mais para conseguir sobreviver na população conforme aumenta-se o valor de $\alpha$.

Para a simulação mostrada na Figura 3, a probabilidade $p$ descrita acima, foi mantida constante em 0.5 .

Como mostrando na figura 4, com o aumento da taxa $\beta$ de infecção e uma taxa $\alpha$ de religação fixa, a taxa $p$, que é a probabilidade com que um vértice não perceba que seu vizinho está infectado, se torna cada vez mais negligenciável. 

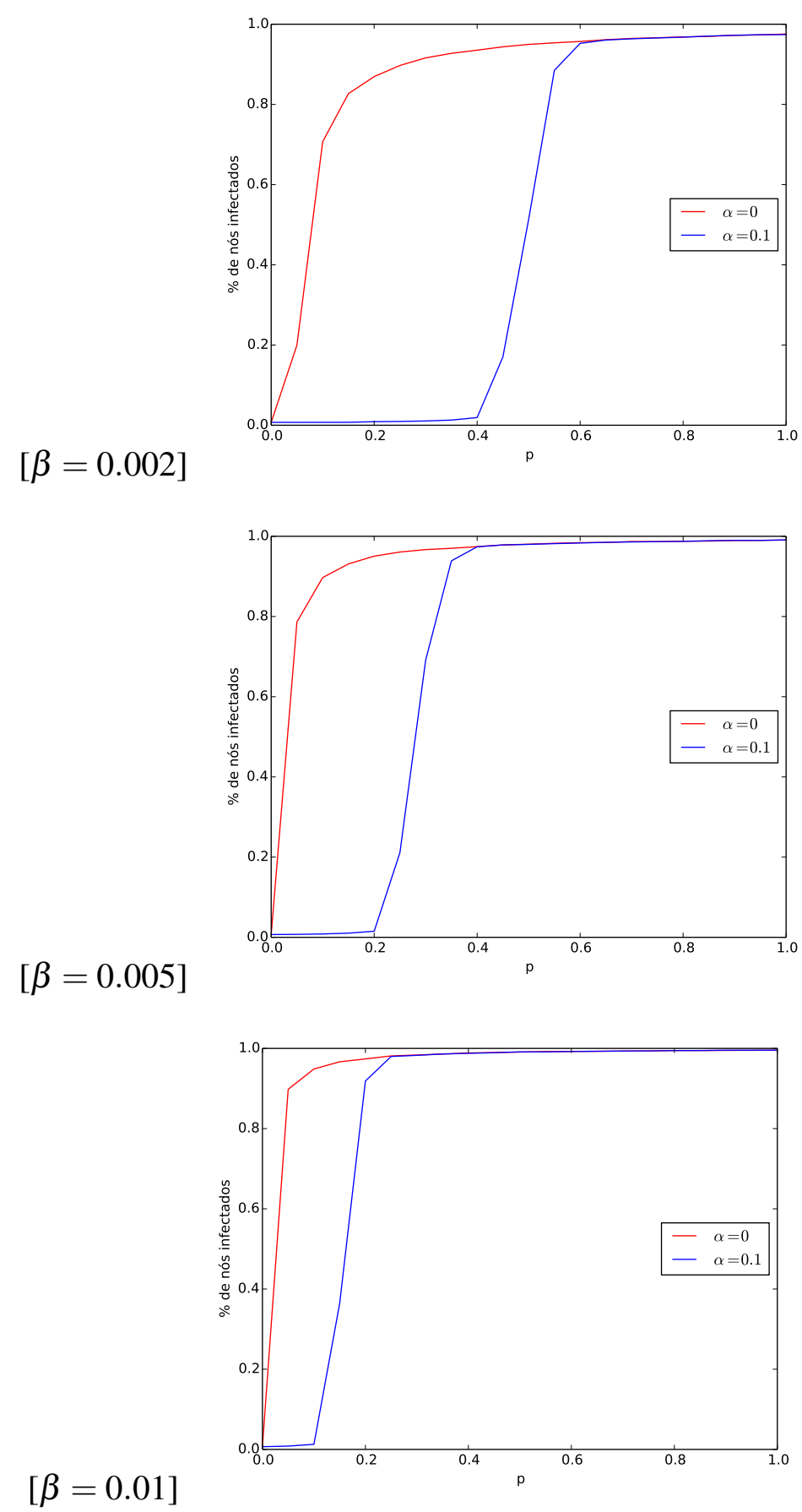

Figura 4 - Comparação entre redes para um $\beta$ fixo.

\subsubsection{Rede BA}

Para o modelo SIS, o efeito que a variação da probabilidade $\alpha$ tem sobre o limiar $\beta_{c}$ não depende da rede utilizada. Em uma rede sem escalas, ou BA, os resultados apresentados são muito similares aos resultados da rede ER. Da mesma forma que na rede aleatória, na rede sem escala o limiar $\beta_{c}$ aumenta conforme aumenta-se $\alpha$. 


\subsection{Propagação da epidemia no modelo SIR}

\subsubsection{Rede BA}

Para a rede sem escala, no caso em que não há religação, a epidemia tende a atingir um ápice de infecção onde grande parte da população torna-se infectada e então, com o passar do tempo, os indivíduos começam a se recuperar (BARABÁSI, 2016). Haja visto que uma vez recuperados eles não podem ser contaminados novamente, a epidemia tende a morrer com a passagem do tempo. Portanto espera-se que o comportamento da infecção para um $\beta$ fixo ao longo do tempo se assemelhe a uma curva em forma de sino. A Figura 5 mostra esse comportamento quando $\alpha=0$

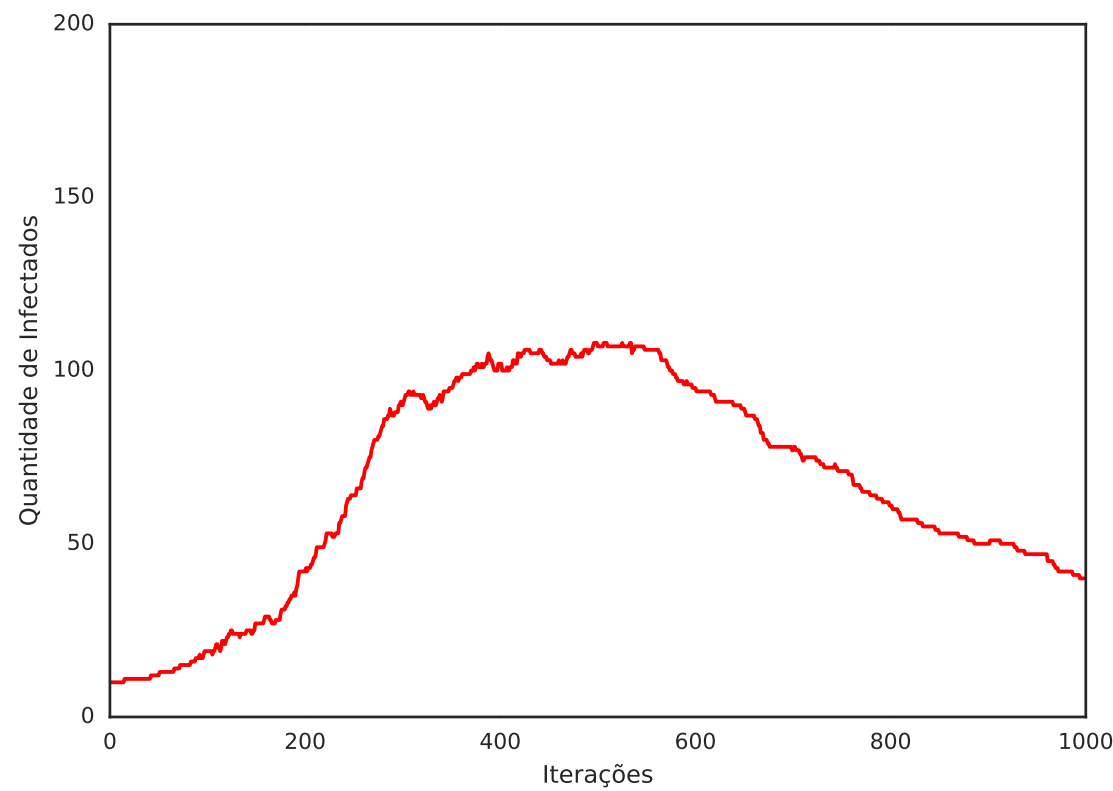

Figura 5 - A infecção começa a crescer até que uma grande parte da população começa a se recuperar, nesse momento a epidemia começa a diminuir. Para essa simulação foi utilizado $\beta=0.0050$

Intuitivamente, imagina-se que quanto maior a taxa $\beta$ de infecção, mais rápido a epidemia tende a atingir seu ápice e isso é realmente comprovado pelas simulações de Monte Carlo. Depois de atingir seu pico em poucas iterações, a epidemia é extinta. A Figura 8 mostra esse comportamento para diferentes $\beta$ 


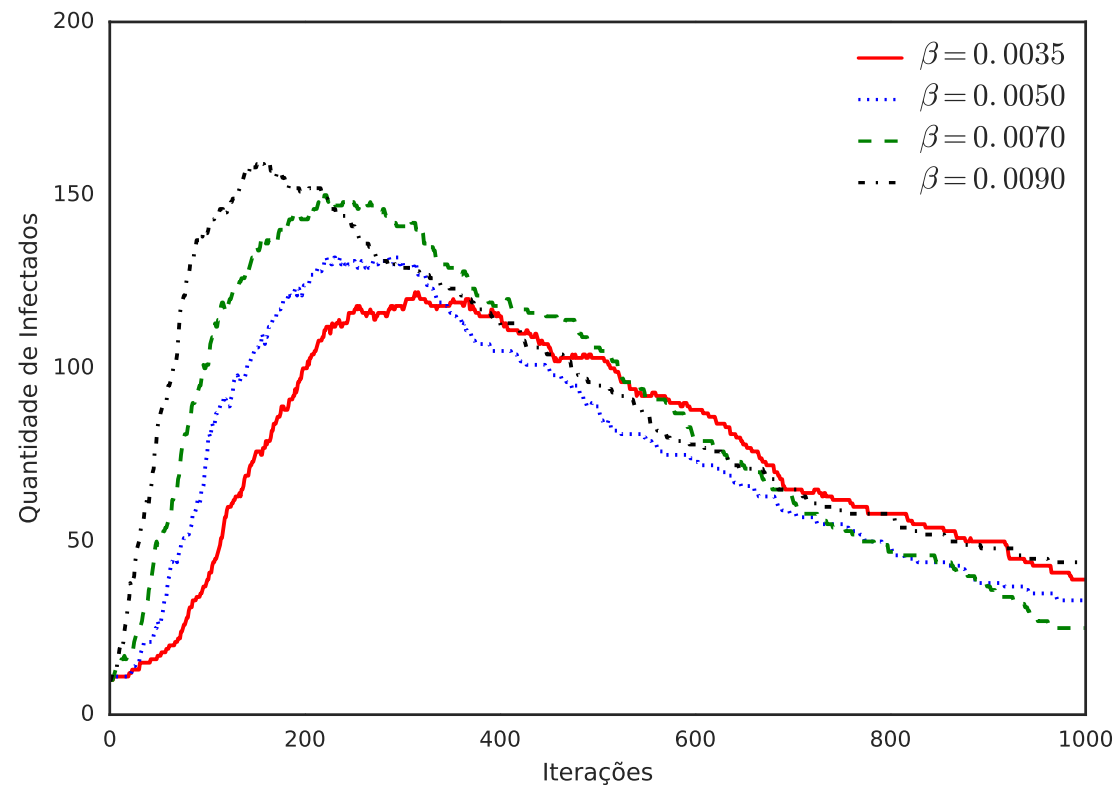

Figura 6 - Evolução da doença ao longo do tempo para diferentes taxas $\beta$. É possível notar que quanto maior o $\beta$ mais rápido a epidemia cresce e consequentemente, mais rápido termina.

Introduzindo a taxa de religação faz com que a epidemia tenha mais dificuldade de atingir seu pico, mas não a impede que a faça para valores maiores de $\beta$. A Figura 7 apresenta uma comparação para valores fixos de $\beta$ entre a mesma rede com e sem religação. 


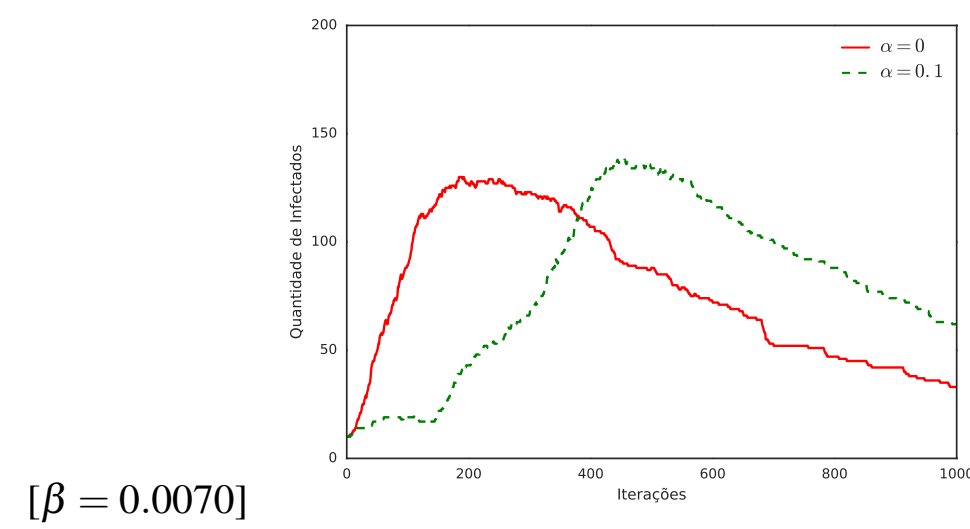

$\beta=0.0070$

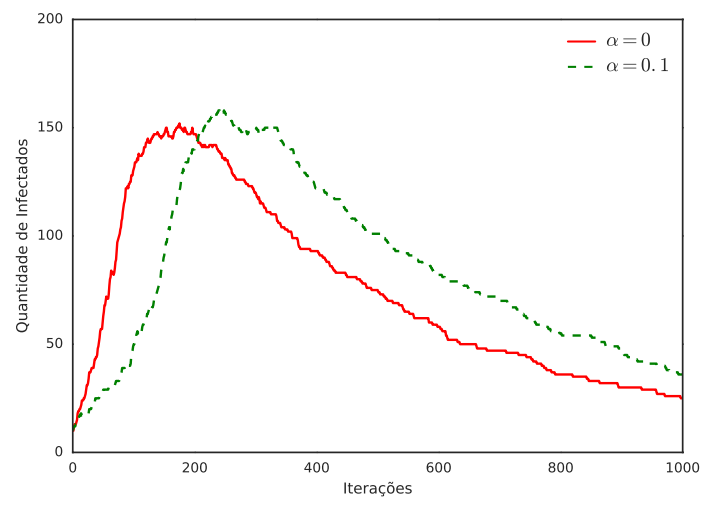

$[\beta=0.0090]$

Figura 7 - Comparação entre a propagação da epidemia com e sem religação.

\subsubsection{Rede ER}

Em redes ER, considerando-se $\alpha=0$, o modelo SIR apresenta crescimento rápido logo nas primeiras iterações e então, como característico do modelo SIR, a epidemia morre aos poucos. A taxa de religação demonstra ter pouco efeito sobre a propagação da doença nesse tipo de rede, sendo necessário um valor alto de $\alpha$ para notar alguma mudança significativa nos resultados. A Figura 8 mostra para diferentes taxas $\alpha$ o estágio da doença ao longo do tempo. 


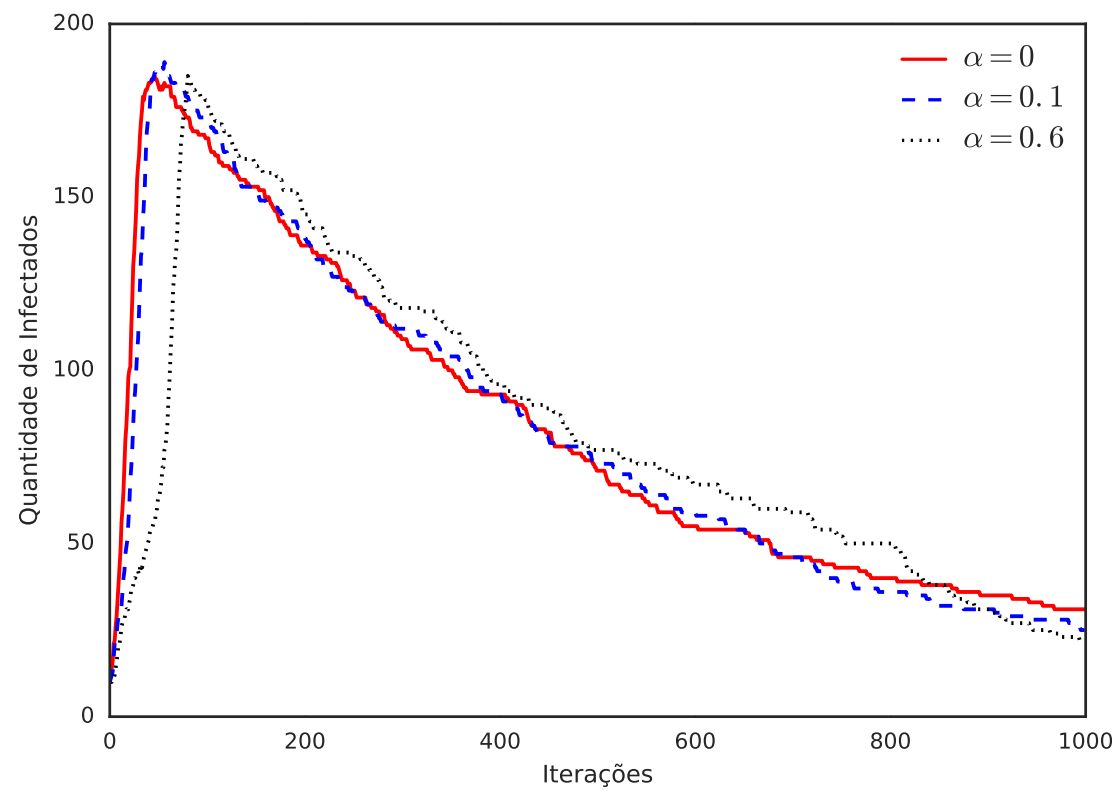

Figura 8 - Modelo SIR executado na rede ER para diferentes taxas de religação. É possível notar que a taxa $\alpha$ tem pouca influencia sobre a rede, sendo necessária altos valores para que a rede demonstre alguma diferença visível no resultado. Para esses experimentos foi utilizado $\beta=0.007$ 



\section{4}

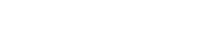

\section{MODELOS EPIDÊMICOS COM MOVIMENTAÇÃO DE AGENTES}

Analisar os padrões resultantes da movimentação de agentes, além de ser um grande desafio, também é vital para algumas áreas do conhecimento tais como: a área de saúde pública (VESPIGNANI, 2009; COLIZZA et al., 2007; BALCAN et al., 2009; KEELING; EAMES, 2005; TOROCZKAI; GUCLU, 2007), o planejamento de cidades (MAKSE; HAVLIN; STANLEY, 1995; HUFNAGEL; BROCKMANN; GEISEL, 2004; ROZENFELD et al., 2008) e engenharia de tráfico (KRINGS et al., 2009; RATTI; RICHENS, 2004). Estudos envolvendo dados de celulares (GONZALEZ; HIDALGO; BARABASI, 2008) e cédulas de dinheiro (BROCKMANN; HUFNAGEL; GEISEL, 2006), indicam que a mobilidade humana pode ser melhor descrita por passeios aleatórios. Um modelo mais complexo de migração aleatória foi proposto por SONG et al.

Neste trabalho, será utilizado um modelo simples de passeio aleatório, iremos observar a dinâmica entre a topologia da rede implícita gerada pela movimentação dos agentes e o comportamento individual dos vértices da rede (YANG; TANG; WANG, 2018). Analisaremos como epidemias e rumores se propagam no modelo proposto e iremos analisar os resultados que emergem dessa dinâmica.

Nesse modelo, $N$ agentes (também chamados de vértices) são distribuídos ao longo de um plano de tamanho $L$ com condições de contorno periódicas. Dois agentes entram em contato se a distância entre eles for menor que $R$ (denominado raio de contato). Uma fração inicial $\rho_{0}$ da população é definida como infectada.

Quando dois agentes entram em contato, eles se tornam ligados e quando se afastam essa ligação é desfeita, isso causa uma constante mudança na topologia.

A posição inicial dos agentes é definida de forma aleatória. A cada passo do algoritmo os agentes decidem se irão se mover ou permanecer no mesmo local. Um vértice se move somente 
se o número de vértices infectados no seu raio de contato for maior que um limiar $E$ definido. Caso isso ocorra, a nova posição do agente é definida pela Equação 4.1

$$
\begin{gathered}
x_{i}(t+1)=x_{i}(t)+v * \cos \left(\theta_{i}(t)\right), \\
y_{i}(t+1)=y_{i}(t)+v * \sin \left(\theta_{i}(t)\right) .
\end{gathered}
$$

onde $x_{i}(t)$ é a posição do individuo no tempo $t, v$ é a velocidade de movimento definida e $\theta_{i}(t)$ é uma variável aleatória uniformemente distribuída no intervalo $[-\pi, \pi]$.

Utilizamos o modelo SIS para simular a propagação de epidemia, de maneira similar aos propostos em (PASTOR-SATORRAS et al., 2015). A cada passo de tempo, um individuo infectado tenta passar sua doença com probabilidade $\beta$ para outro suscetível que esteja dentro do seu raio de contato. Ao mesmo tempo, indivíduos infectados também tentam se recuperar a uma taxa $\mu$

Utilizamos o modelo proposto por Maki and Thompson (Modelo MT) (CASTELLANO; FORTUNATO; LORETO, 2009) para propagação de rumores. O processo começa com um informante e $N-1$ ignorantes, onde $N$ é o número de nós na rede. A cada passo de tempo, informantes tentam passar o rumor para seus vizinhos ignorantes a uma taxa $\beta$. Por outro lado informantes que tenham contato com outros informantes ou contidos tornam-se contidos a uma taxa $\mu$

\subsection{Propagação da epidemia no modelo SIS}

Uma analise fundamental em modelos SIS é a do limiar da infecção $\beta_{c}$. Taxas de infecções abaixo desse limiar fazem com que a infecção não perdure na população (BOGUNÁ; PASTOR-SATORRAS, 2002; PARSHANI; CARMI; HAVLIN, 2010; CASTELLANO; PASTOR-SATORRAS, 2010; BOGUNÁ; CASTELLANO; PASTOR-SATORRAS, 2013).

Para valores grandes do limiar $E$, sendo $E$ o número minimo de infectados na vizinhança necessários para que um agente decida se mover, os indivíduos não se movem e tem menos chances de escapar da infecção. Para valores muito pequenos, os agentes se movem a todo momento em direções aleatórias, o sistema tende ao sistema homogêneo onde cada individuo tem a mesma chance de ser infectado. Logo, tanto valores baixos quanto altos do limiar $E$ fazem com que a epidemia consiga perdurar no sistema. Para valores medianos, alguns agentes conseguem escapar da epidemia e se isolam em regiões onde a concentração de doentes é menor. Dessa forma, a epidemia tem menor probabilidade de atingir esses indivíduos e acaba desaparecendo com o tempo. Portanto existe um valor ótimo $E_{o p t}$ em que o valor $\beta_{c}$ atinge seu máximo. 


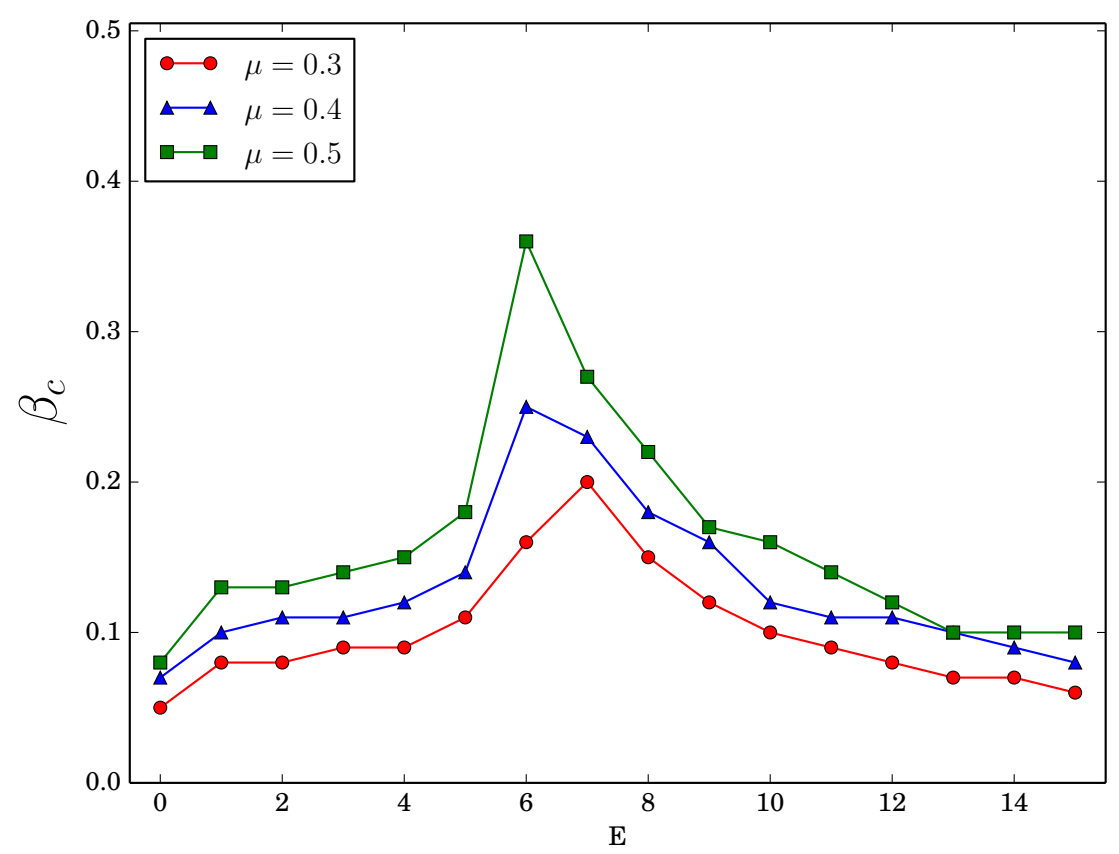

Figura 9 - Comparação entre a taxa critica de infecção e o valor minimo para que os agentes se movam. Podemos notar que existe um ponto onde a taxa critica atinge seu máximo. Foram utilizados três valores diferentes da taxa de recuperação $\mu$. A velocidade utilizada foi de $V=0.3$. Raio de contato $r=0.6$. Desvio padrão médio para o calculo dos $\beta_{c}$ de 0.000130

Como podemos notar na Figura 14, quanto mais aumentamos a taxa de recuperação $\mu$, mais o valor critico $E_{o p t}$ cresce. Isso ocorre pois quanto mais rápido a população consegue se recuperar, mais dificuldade a epidemia tem de se espalhar no sistema.

Agentes com mais vizinhos possuem maior probabilidade de serem infectados, então tais indivíduos tendem a sair e mover-se para regiões menos populadas. Por outro lado, agentes com menos vizinhos são mais prováveis de continuar no mesmo lugar pois tendem a ter menos que $E$ vizinhos infectados. Portanto o número médio de vizinhos $<k>$ de um vértice diminui com o tempo, como pode ser notado na Figura 10. 
[a]
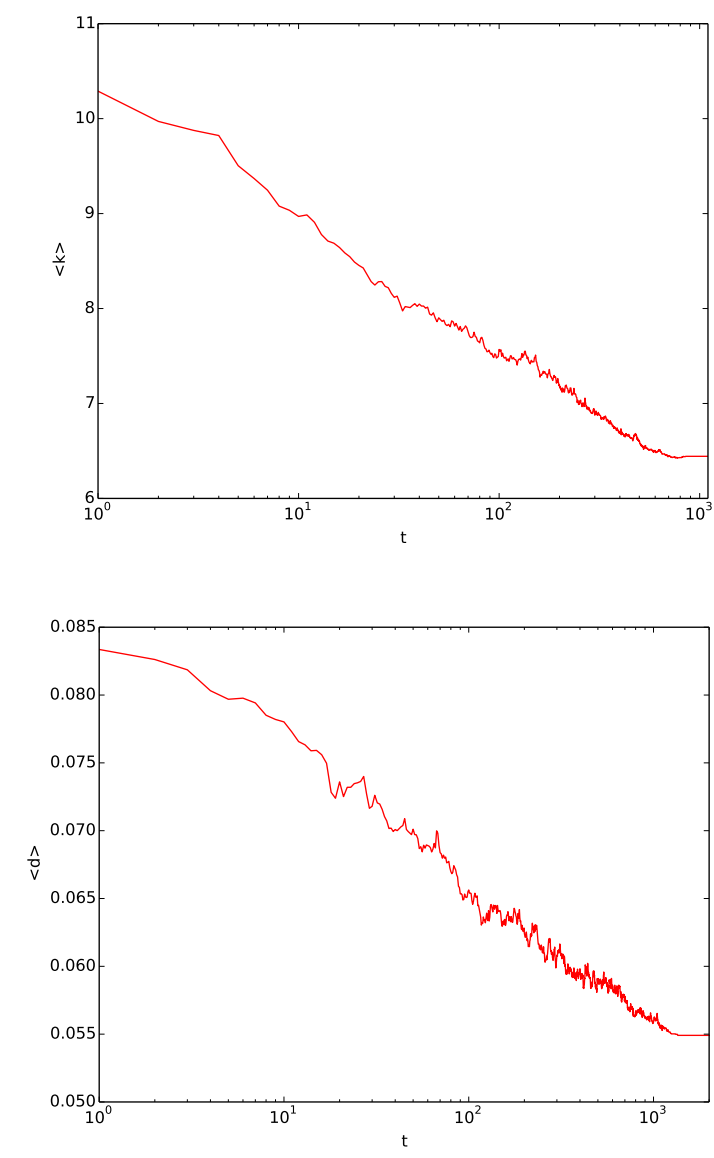

Figura 10 - (a) Número médio $<k>$ de vizinhos de um nó diminui com o tempo. (b) Distância média $<d>$ entre dois vértices vizinhos aumenta de acordo com a passagem do tempo $t$. Foram utilizados os seguintes parâmetros: Taxa de infecção $\beta=0.15$, taxa de recuperação $\mu=0.4$, raio de contato $r=0.45$, velocidade de movimento $v=0.3$ e limiar de movimento $E=7$. Gráficos na escala logarítmica. Desvio padrão para o calculo de (a) 0.000158. Desvio padrão para o calculo de (b) 0.000095

Contra-intuitivamente, o decrescimento do número médio de vizinhos $<k>$ não aumenta a distância média entre dois agentes. Na Figura 10 é possível notar que a distância média entre dois agentes vizinhos $\langle d>$ diminui quando aumentamos $t$ para valores moderados de $E$.

A diminuição do número médio de vizinhos e da distância média entre dois agentes indica que os indivíduos estão se agrupando em locais isolados uns dos outros. Na Figura 11 vemos o aumento do número de componentes conexas e a diminuição do tamanho da maior componente. Isso ocorre pois as áreas em que os agentes se agrupam estão longe uma das outras. 
[a]

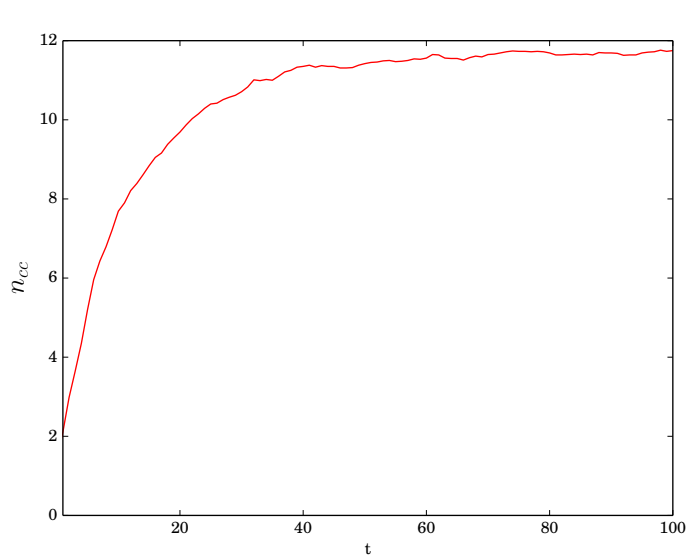

]

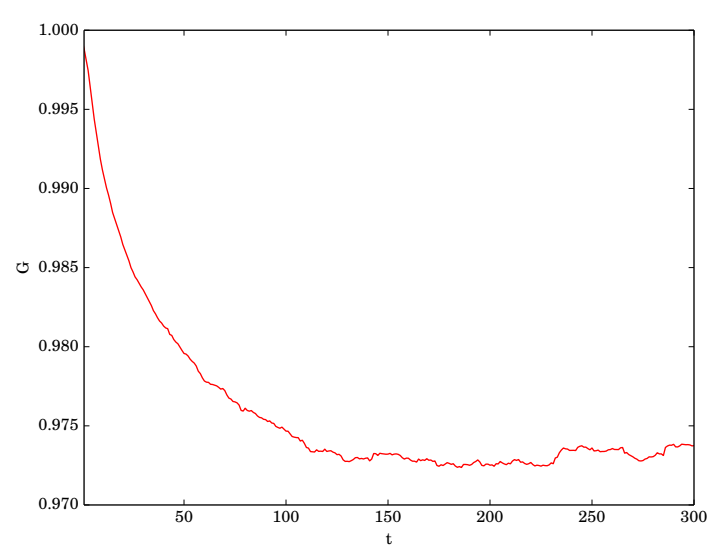

[b]

Figura 11 - (a) Número de componentes conexas ao longo do tempo. (b) Porcentagem de indivíduos presentes na maior componente conexa ao longo do tempo. Foram utilizados os seguintes parâmetros: Taxa de propagação do rumor $\beta=0.35$, taxa de recuperação $\mu=0.1$, raio de contato $r=0.45$, velocidade de movimento $v=0.3$, limiar $E=7$. Desvio padrão para o calculo de (a) 0.000158. Desvio padrão para o calculo de (b) 0.000348

Combinando os resultados da Figura 10 e da Figura 11 é possível entender porque valores médios de $E$ levam a epidemia a atingir seu maior $\beta_{c}$. Como podemos ver, para valores médios, os agentes tendem a formar pequenas comunidades isoladas que dificultam a passagem da epidemia.

O aumento da densidade de agentes no plano, faz com que a porcentagem de infectados cresça exponencialmente, o mesmo pode ser dito para o tamanho da área de contato, como pode ser visto na Figura 12 


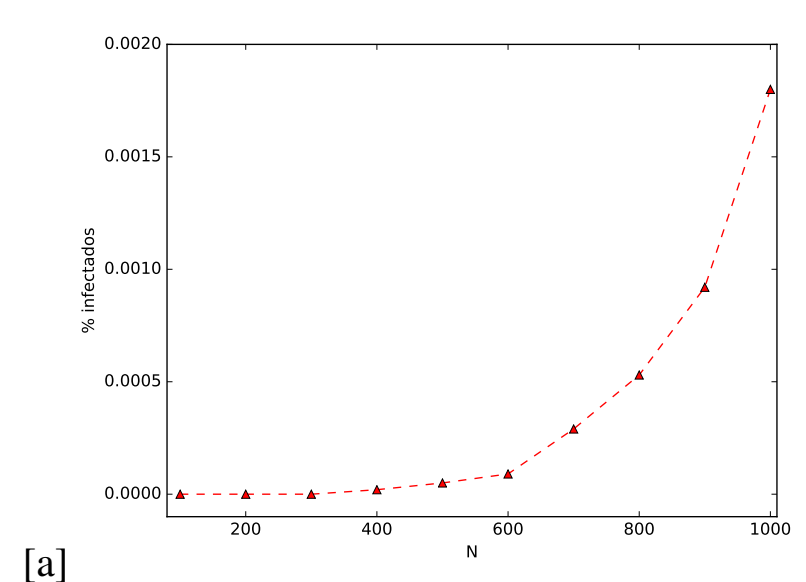

[a]

[b]

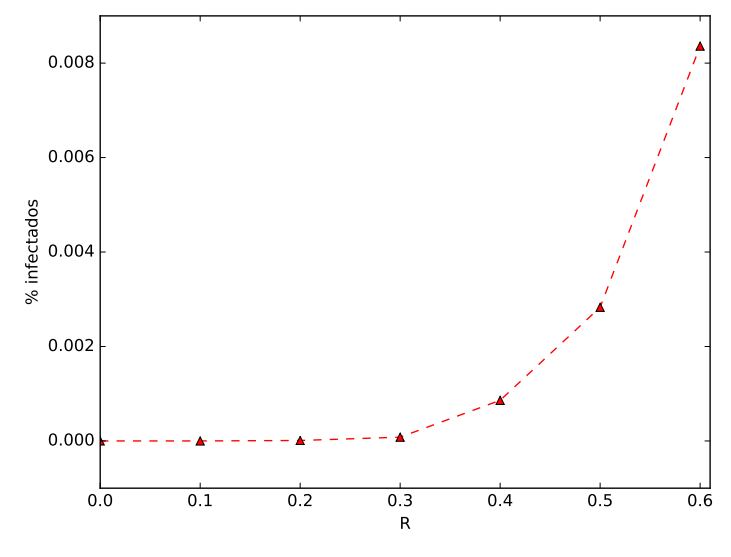

Figura 12 - (a) Número de agentes versus porcentagem de infectados. É possível notar que a porcentagem de infectados cresce exponencialmente com o aumento do número de agentes (b) Raio de contato versus porcentagem de infectados. O mesmo efeito que ocorreu para a densidade de agentes também ocorre para a variação do raio de contato. Foram utilizados os seguintes parâmetros: Taxa de propagação do rumor $\beta=0.35$ (Quando não especificado), taxa de recuperação $\mu=0.1$, raio de contato $r=0.45$, velocidade de movimento $v=0.3, E=2$ (Quando não especificado). Desvio Padrão de (a) 0.000474. Desvio padrão de (b) 0.000032

Um fato interessante ocorre quando variamos a velocidade dos agentes e analisamos seu impacto na porcentagem de infectados. Quando a velocidade está próxima a zero, a infecção tem certa facilidade para se propagar na população, isso se da ao fato de que agentes suscetíveis não tem como fugir. Porém quando a velocidade começa a crescer é possível perceber uma certa constante na porcentagem de infectados, que cai drasticamente em comparação a quando os agentes não se movimentavam. Uma possível explicação para esse fato é que como os agentes se movimentam todos com a mesma velocidade, o fato de os suscetíveis se moverem mais rápido não facilita a fuga da epidemia, pois os infectados também se movem mais rápido. Esse efeito pode ser visto na Figura 13. 


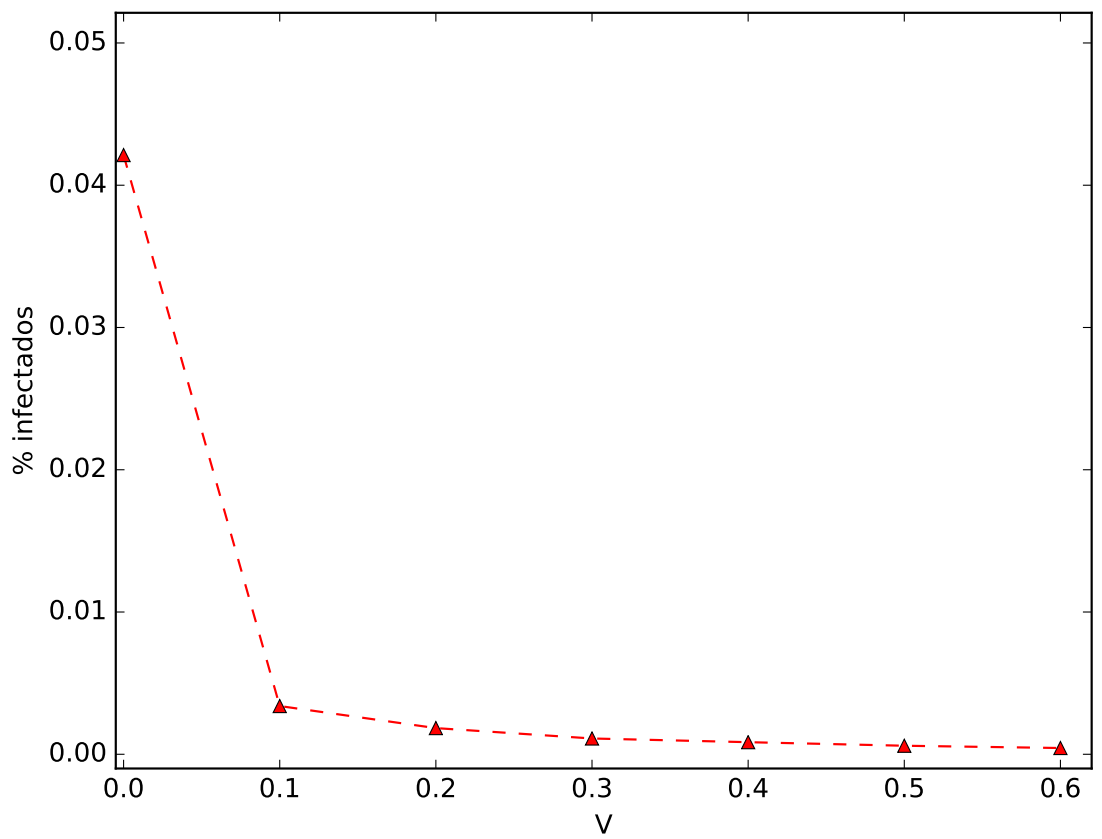

Figura 13 - Velocidade dos agentes versus $\%$ de infectados. O Raio de contato utilizado foi $r=0.45$, Taxa de propagação do rumor $\beta=0.35$, taxa de recuperação $\mu=0.3$ Desvio padrão de 0.000221 



\section{EPIDEMIAS EM MEIOS HETEROGÊNEOS}

\subsection{Fractal Landscape}

Fractal Landscapes são superfícies geradas apartir de algum processo estocástico que resulta em terrenos aleatórios que se parecem com superfícies reais. Nesse trabalho o terreno foi construído com o método de movimento Browniano (MANDELBROT; NESS, 1968). O movimento Browniano é uma generalização de um processo aleatório $X(t)$ que possuí incrementos Gaussianos tal que:

$$
\operatorname{var}\left(X\left(t_{2}\right)-X\left(t_{1}\right)\right) \propto\left|t_{2}-t_{1}\right|^{2 H},
$$

onde var representa a variância e $\mathrm{H}$ é o expoente de Hurst no intervalo $[0,1]$. Quando $H$ é próximo de 0 , o algoritmo produz superfícies com muitos relevos e quanto mais $H$ se aproxima de 1 , mais suave o relevo vai se tornando.

\subsection{Simulação}

As simulações continuaram a ser realizadas da forma explicada no capítulo 4, com a diferença de que nesse novo modelo a velocidade dos agentes é computada considerando-se a gravidade. Dessa forma, a ação da força da gravidade faz com que seja difícil a travessia dos agentes por meios que apresentam subidas e decidas com maior angulação. $\mathrm{O}$ calculo do angulo do plano em que o agente se encontra em relação a superfície é dado pelo angulo entre os vetores normais:

$$
\theta=\arcsin \frac{|\vec{n} \cdot \vec{u}|}{|\vec{n}| \cdot|\vec{u}|}
$$

onde $\vec{n}$ é o vetor normal ao plano para onde o agente esta se locomovendo e $\vec{u}$ é o vetor normal ao plano $x y$. Seja a aceleração da gravidade no eixo x em um plano inclinado $a_{x}=g * \sin (\theta)$ onde $g$ é a aceleração da gravidade e $\theta$ é o angulo do plano em relacão à superfície. A nova 
velocidade do agente então é:

$$
V_{\text {new }}= \begin{cases}V_{\text {ini }}-a_{x}, & \text { se o individuo está subindo } \\ V_{\text {ini }}+a_{x}, & \text { se o individuo esta descendo }\end{cases}
$$

onde $V_{i n i}$ é a aceleração inicial definida por parâmetro e $a_{x}$ é a aceleração da gravidade no eixo $x$

\subsubsection{Propagação de epidemia no modelo SIS}

No modelo SIS, é possível observar que o relevo exerce influência significativa sobre o valor ótimo de pessoas que o agente deve esperar estar contaminadas antes de se mover, ou $E_{o p t}$, tendo visto que o valor muda para diferentes valores do coeficiente de Hurst $H$.

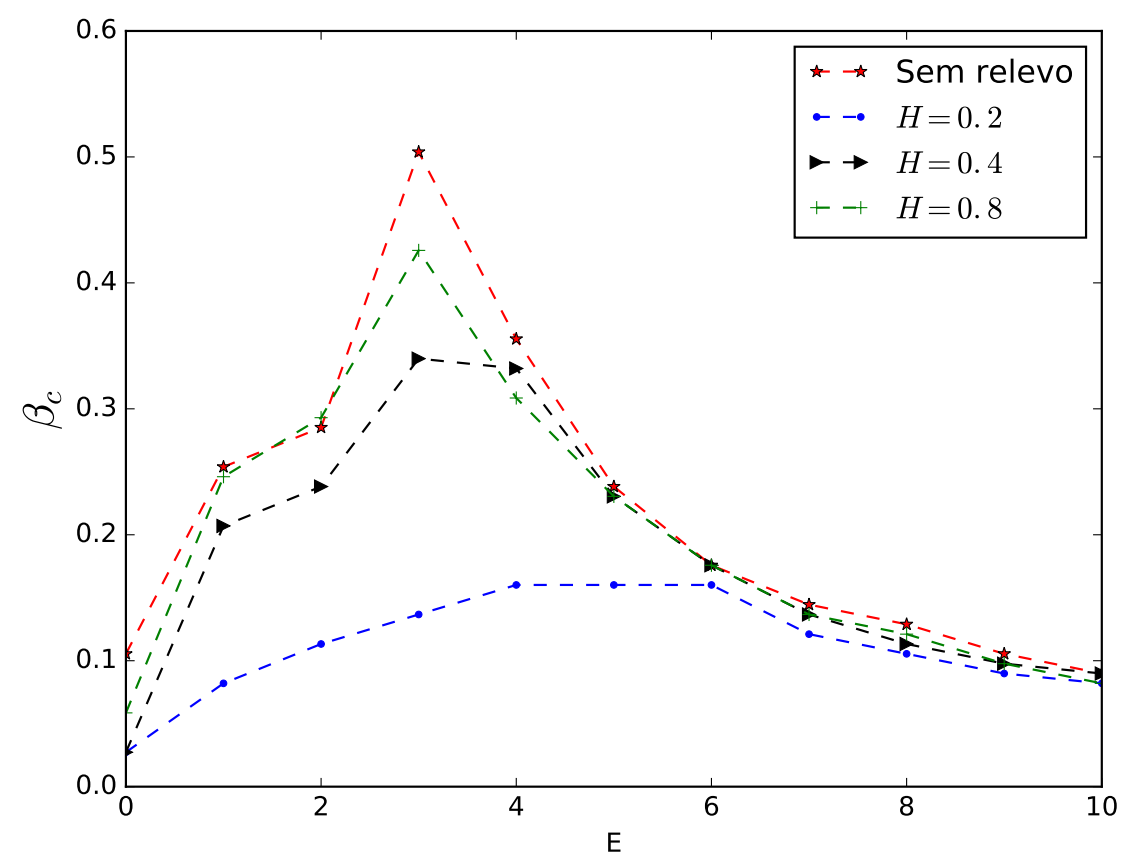

Figura 14 - Comparação da taxa $E_{\text {opt }}$ para diferentes valores de $H$. A taxa de recuperação $\mu$ utilizada foi de 0.3. A velocidade utilizada foi de $V=0.3$. Raio de contato $r=0.6$. Desvio padrão médio (Soma dos desvios padrões para cada $\mathrm{H}$ ) 0.000063

Pela figura 15 nota-se que a porcentagem de indivíduos infectados ao final do processo também muda bastante para diferentes valores de $H$. Nota-se que para valores menores do coeficiente, ou seja, para superfícies com relevos mais íngremes, a porcentagem de infectados ao final do processo é maior quando comparado a valores maiores do coeficiente de Hurst $H$. 


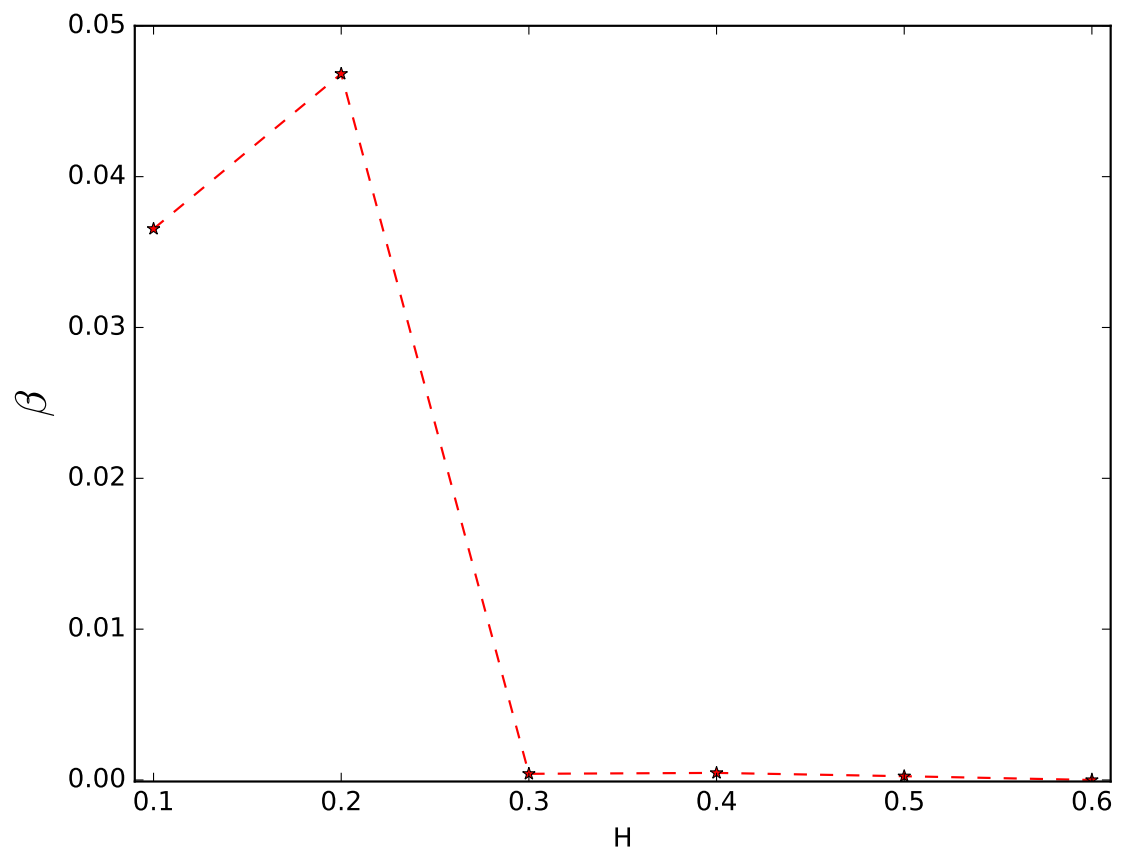

Figura 15 - Comparação da porcentagem de infectados ao final do processo para diferentes valores de $H$. A taxa de recuperação $\mu$ utilizada foi de 0.3 . A velocidade utilizada foi de $V=0.3$. Raio de contato $r=0.6$ Desvio padrão 0.000158

Vale notar também que conforme o coeficiente cresce, ou seja, conforme o plano perde seus relevos, mais dificuldade a epidemia tem de sobreviver na população, eventualmente chegando a zero para valores grandes de $H$.

Vales mais profundos e picos mais íngremes são fatores que influenciam esses resultados, como pode ser visto na Figura 16. 


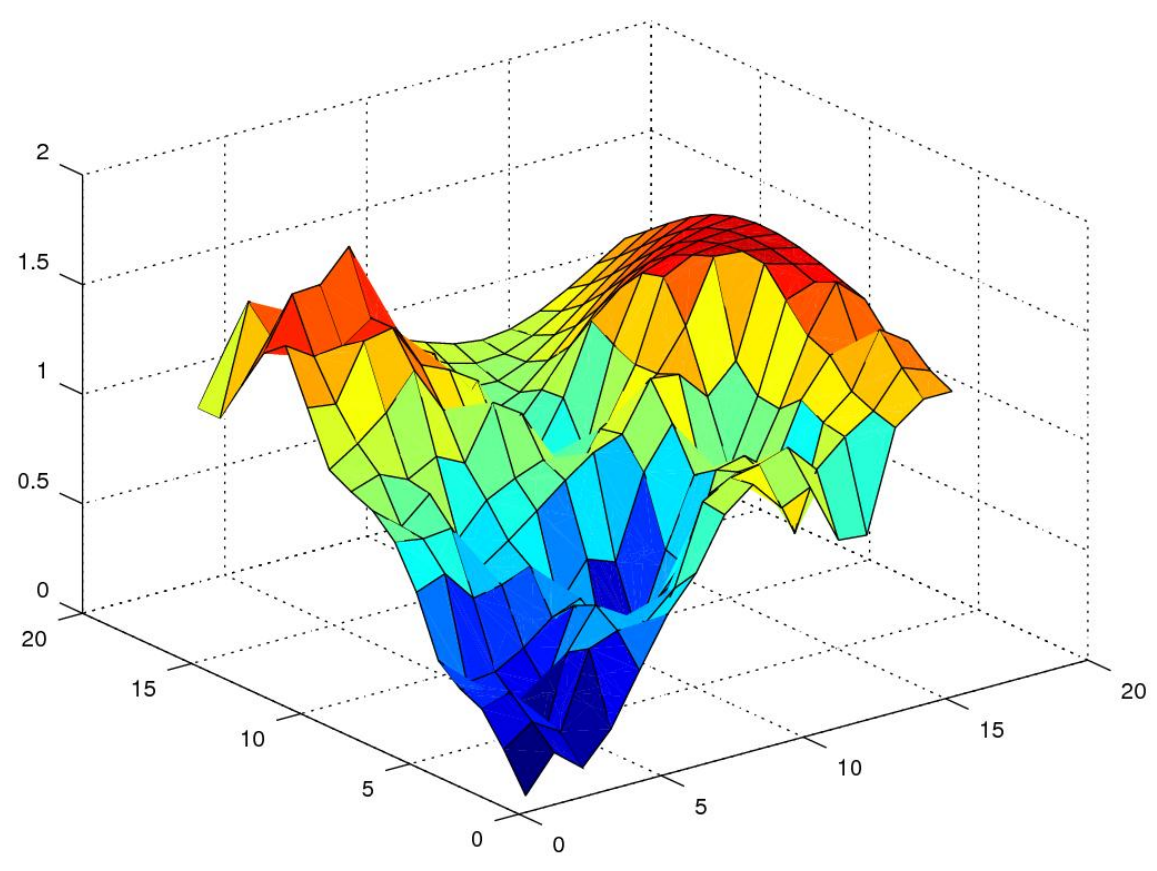

(a) Mapa de alturas, quanto mais perto do azul mais baixo e quanto mais perto do vermelho mais em cima o plano se encontra

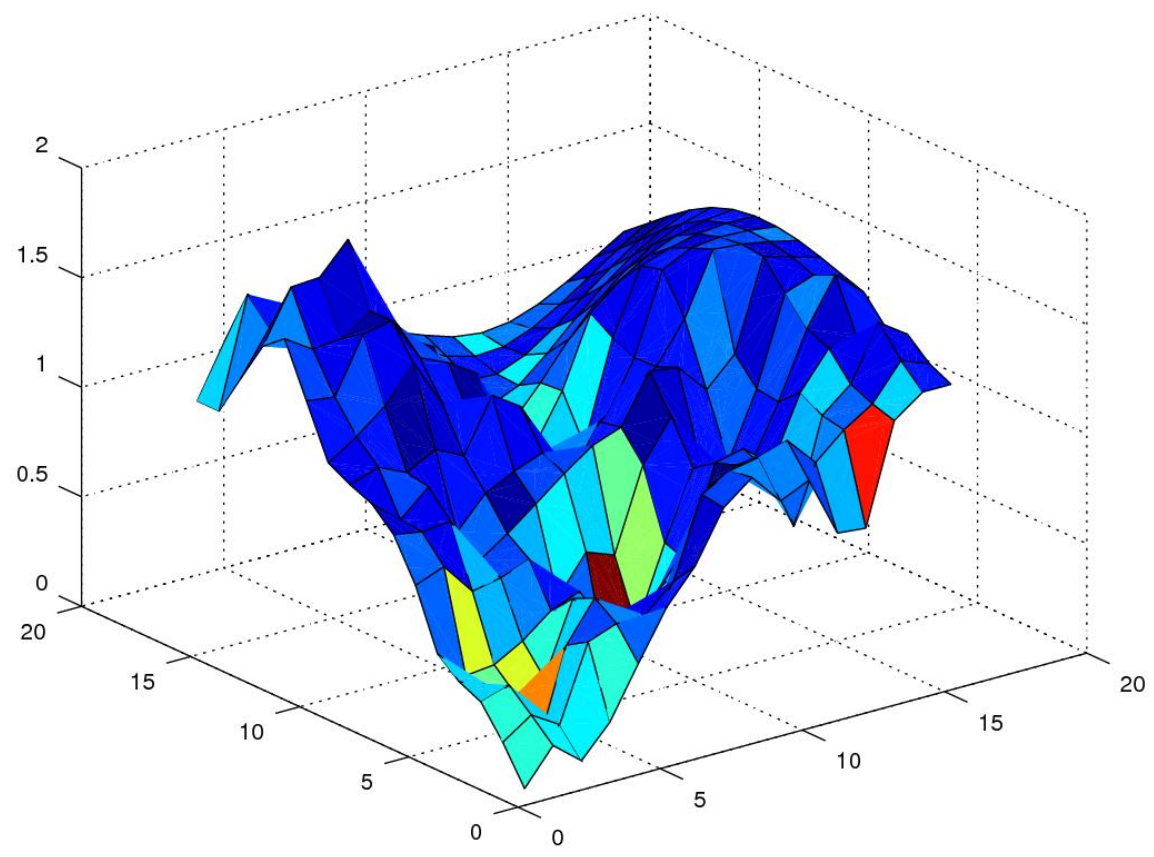

(b) Mapa de infectados, quanto mais vermelho mais infectados existem naquela área, e quanto mais perto do azul menos infectados existem

Figura 16 - A taxa de recuperação $\mu$ utilizada foi de 0.3 . A velocidade utilizada foi de $V=0.3$. Raio de contato $r=0.6$ Desvio padrão 0.000370 
Como pode-se notar pela figura 17(a), as partes mais baixas, representadas pelas cores mais azuladas tendem a atrair mais indivíduos, que acabam ficando presos ali. A figura 17(b) mostra que a maioria dos infectados acabaram nesses vales.

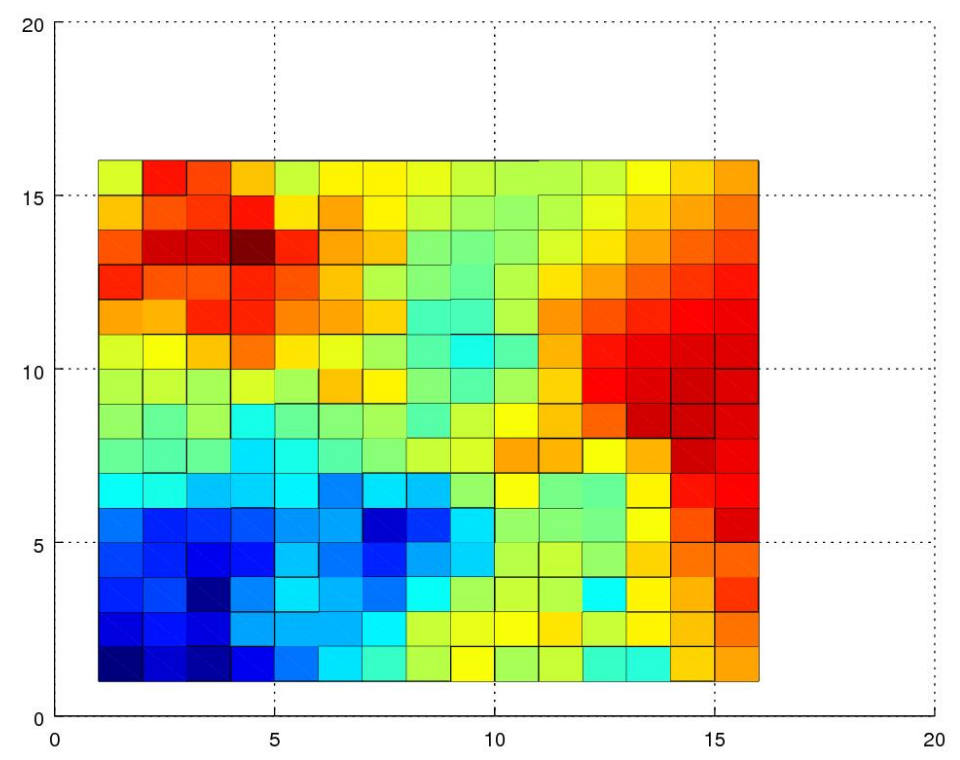

(a) Mapa de alturas planar, quanto mais perto do azul mais baixo e quanto mais perto do vermelho mais em cima o plano se encontra

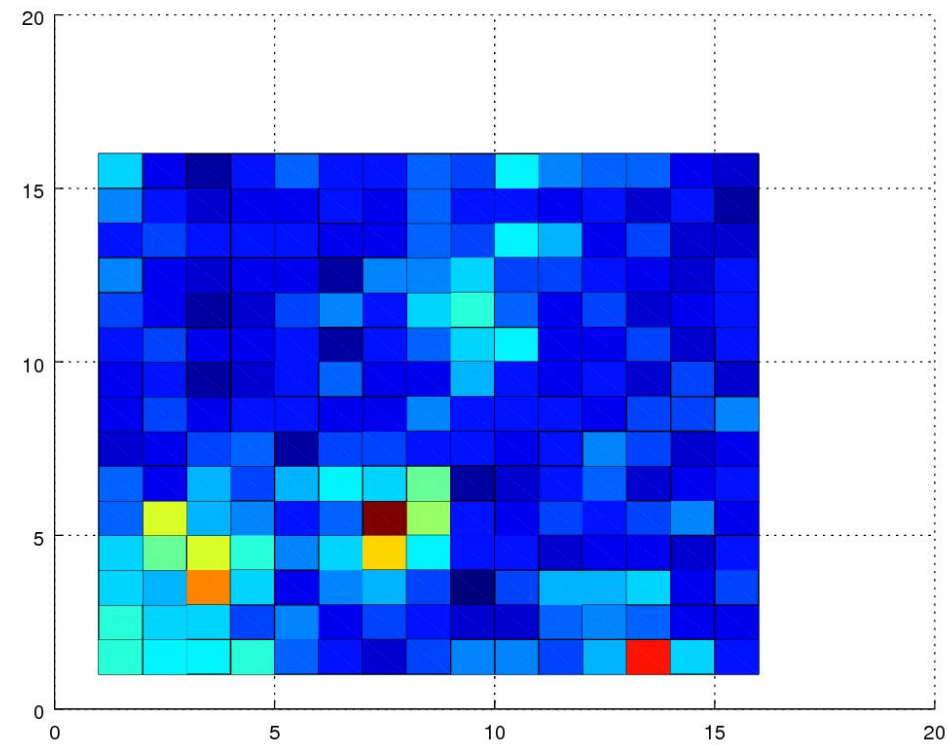

(b) Mapa de infectados planar, quanto mais vermelho mais infectados existem naquela área, e quanto mais perto do azul menos infectados existem

Figura 17 - A taxa de recuperação $\mu$ utilizada foi de 0.3 . A velocidade utilizada foi de $V=0.3$. Raio de contato $r=0.6$ Desvio padrão 0.000370 



\section{TRABALHOS FUTUROS}

\subsection{Conclusão e trabalhos Futuros}

Nesse trabalho foi introduzido um novo modelo de movimentação de agentes que traz um novo parâmetro quando comparado com outros modelos de movimentação de agentes já existentes na literatura, o número $E$ minimo de agentes infectados que os indivíduos suscetíveis devem esperar estar contaminados para começar a se movimentar. Após os experimentos terem sido realizados, foi descoberto que existe um valor ótimo $E_{o p t}$ que os agentes devem esperar antes de se mover para que a epidemia tenha mais dificuldades para se propagar.

Outro resultado interessante é que o modelo sofre influencia direta do meio em que os agentes estão se movimentando, sendo que meios com mais relevos facilitam a propagação da epidemia.

Desafios futuros incluem desenvolver o mesmo modelo para propagação de rumores; mudar a estratégia de movimentação pois no modelo atual a movimentação é puramente aleatória; e testar diferentes tipos de relevos, pois nesse trabalho só foi utilizado o método de movimento Browniano. Também é necessária uma analise mais aprofundada de como o relevo afeta a propagação da epidemia. 



\section{REFERÊNCIAS}

ALBERT, R.; JEONG, H.; BARABÁSI, A.-L. Error and attack tolerance of complex networks. Nature, Nature Publishing Group, v. 406, n. 6794, p. 378-382, 2000. Citado na página 19.

BALCAN, D.; HU, H.; GONCALVES, B.; BAJARDI, P.; POLETTO, C.; RAMASCO, J. J.; PAOLOTTI, D.; PERRA, N.; TIZZONI, M.; BROECK, W. Van den et al. Seasonal transmission potential and activity peaks of the new influenza a $(\mathrm{h} 1 \mathrm{n} 1)$ : a monte carlo likelihood analysis based on human mobility. BMC medicine, BioMed Central, v. 7, n. 1, p. 45, 2009. Citado na página 39.

BARABÁSI, A.-L. Network science. [S.1.]: Citeseer, 2016. Citado nas páginas 20, 23, 24 e 34.

BARRAT, A.; BARTHELEMY, M.; VESPIGNANI, A. Dynamical processes on complex networks. [S.1.]: Cambridge University Press, 2008. Citado na página 19.

BARTHÉLEMY, M. Spatial networks. Physics Reports, Elsevier, v. 499, n. 1, p. 1-101, 2011. Citado na página 24.

BOCCALETTI, S.; LATORA, V.; MORENO, Y.; CHAVEZ, M.; HWANG, D.-U. Complex networks: Structure and dynamics. Physics reports, Elsevier, v. 424, n. 4, p. 175-308, 2006. Citado nas páginas 19 e 23.

BOGUNÁ, M.; CASTELLANO, C.; PASTOR-SATORRAS, R. Nature of the epidemic threshold for the susceptible-infected-susceptible dynamics in networks. Physical review letters, APS, v. 111, n. 6, p. 068701, 2013. Citado na página 40.

BOGUNÁ, M.; PASTOR-SATORRAS, R. Epidemic spreading in correlated complex networks. Physical Review E, APS, v. 66, n. 4, p. 047104, 2002. Citado na página 40.

BORNHOLDT, S.; ROHLF, T. Topological evolution of dynamical networks: Global criticality from local dynamics. Physical Review Letters, APS, v. 84, n. 26, p. 6114, 2000. Citado na página 30.

BRAUER, F. Compartmental models in epidemiology. In: Mathematical epidemiology. [S.l.]: Springer, 2008. p. 19-79. Citado na página 24.

BROCKMANN, D.; HUFNAGEL, L.; GEISEL, T. The scaling laws of human travel. arXiv preprint cond-mat/0605511, 2006. Citado na página 39.

CASTELLANO, C.; FORTUNATO, S.; LORETO, V. Statistical physics of social dynamics. Reviews of modern physics, APS, v. 81, n. 2, p. 591, 2009. Citado na página 40.

CASTELLANO, C.; PASTOR-SATORRAS, R. Thresholds for epidemic spreading in networks. Physical review letters, APS, v. 105, n. 21, p. 218701, 2010. Citado na página 40.

CATANZARO, M.; BOGUNÁ, M.; PASTOR-SATORRAS, R. Generation of uncorrelated random scale-free networks. Physical Review E, APS, v. 71, n. 2, p. 027103, 2005. Citado na página 29. 
COLIZZA, V.; BARRAT, A.; BARTHÉLEMY, M.; VESPIGNANI, A. Predictability and epidemic pathways in global outbreaks of infectious diseases: the sars case study. BMC medicine, BioMed Central, v. 5, n. 1, p. 34, 2007. Citado na página 39.

COSTA, L. d. F.; JR, O. N. O.; TRAVIESO, G.; RODRIGUES, F. A.; BOAS, P. R. V.; ANTIQUEIRA, L.; VIANA, M. P.; ROCHA, L. E. C. Analyzing and modeling real-world phenomena with complex networks: a survey of applications. Advances in Physics, Taylor \& Francis, v. 60, n. 3, p. 329-412, 2011. Citado na página 19.

DALEY, D.; KENDALL, D. G. Stochastic rumours. IMA Journal of Applied Mathematics, IMA, v. 1, n. 1, p. 42-55, 1965. Citado na página 26.

ERDOS, P.; RÉNYI, A. On the evolution of random graphs. Publ. Math. Inst. Hung. Acad. Sci, v. 5, n. 1, p. 17-60, 1960. Citado na página 23.

GONZALEZ, M. C.; HIDALGO, C. A.; BARABASI, A.-L. Understanding individual human mobility patterns. arXiv preprint arXiv:0806.1256, 2008. Citado na página 39.

GROSS, T.; BLASIUS, B. Adaptive coevolutionary networks: a review. Journal of The Royal Society Interface, The Royal Society, v. 5, n. 20, p. 259-271, 2008. Citado nas páginas 19 e 21.

GROSS, T.; D'LIMA, C. J. D.; BLASIUS, B. Epidemic dynamics on an adaptive network. Physical review letters, APS, v. 96, n. 20, p. 208701, 2006. Citado nas páginas 21, 30 e 31.

HOLME, P.; GHOSHAL, G. Dynamics of networking agents competing for high centrality and low degree. Physical review letters, APS, v. 96, n. 9, p. 098701, 2006. Citado na página 30.

HOLME, P.; NEWMAN, M. E. Nonequilibrium phase transition in the coevolution of networks and opinions. Physical Review E, APS, v. 74, n. 5, p. 056108, 2006. Citado na página 30.

HUFNAGEL, L.; BROCKMANN, D.; GEISEL, T. Forecast and control of epidemics in a globalized world. Proceedings of the National Academy of Sciences of the United States of America, National Acad Sciences, v. 101, n. 42, p. 15124-15129, 2004. Citado na página 39.

ITO, J.; KANEKO, K. Spontaneous structure formation in a network of chaotic units with variable connection strengths. Physical Review Letters, APS, v. 88, n. 2, p. 028701, 2001. Citado na página 30 .

KEELING, M. J.; EAMES, K. T. Networks and epidemic models. Journal of the Royal Society Interface, The Royal Society, v. 2, n. 4, p. 295-307, 2005. Citado na página 39.

KRINGS, G.; CALABRESE, F.; RATTI, C.; BLONDEL, V. D. Urban gravity: a model for intercity telecommunication flows. Journal of Statistical Mechanics: Theory and Experiment, IOP Publishing, v. 2009, n. 07, p. L07003, 2009. Citado na página 39.

MAKSE, H. A.; HAVLIN, S.; STANLEY, H. E. Modelling urban growth patterns. Nature, Springer, v. 377, n. 6550, p. 608-612, 1995. Citado na página 39.

MANDELBROT, B. B.; NESS, J. W. V. Fractional brownian motions, fractional noises and applications. SIAM review, SIAM, v. 10, n. 4, p. 422-437, 1968. Citado na página 47.

NEKOVEE, M.; MORENO, Y.; BIANCONI, G.; MARSILI, M. Theory of rumour spreading in complex social networks. Physica A: Statistical Mechanics and its Applications, Elsevier, v. 374, n. 1, p. 457-470, 2007. Citado na página 27. 
NEWMAN, M. Networks: an introduction. [S.1.]: Oxford University Press, 2010. Citado nas páginas 15 e 26.

PARSHANI, R.; CARMI, S.; HAVLIN, S. Epidemic threshold for the susceptible-infectioussusceptible model on random networks. Physical review letters, APS, v. 104, n. 25, p. 258701, 2010. Citado na página 40.

PASTOR-SATORRAS, R.; CASTELLANO, C.; MIEGHEM, P. V.; VESPIGNANI, A. Epidemic processes in complex networks. Rev. Mod. Phys., American Physical Society, v. 87, p. 925-979, Aug 2015. Disponível em: <http://link.aps.org/doi/10.1103/RevModPhys.87.925>. Citado nas páginas 19, 21, 25, 26, 29 e 40.

PASTOR-SATORRAS, R.; VESPIGNANI, A. Epidemic dynamics and endemic states in complex networks. Physical Review E, APS, v. 63, n. 6, p. 066117, 2001. Citado na página 31.

Epidemic spreading in scale-free networks. Physical Review Letters, APS, v. 86, n. 14, p. 3200, 2001. Citado na página 19.

RATTI, C.; RICHENS, P. Raster analysis of urban form. Environment and Planning B: Planning and Design, SAGE Publications Sage UK: London, England, v. 31, n. 2, p. 297-309, 2004. Citado na página 39.

RODRIGUES, F. A.; PERON, T. K. D.; JI, P.; KURTHS, J. The kuramoto model in complex networks. Physics Reports, Elsevier, 2015. Citado na página 19.

ROZENFELD, H. D.; RYBSKI, D.; ANDRADE, J. S.; BATTY, M.; STANLEY, H. E.; MAKSE, H. A. Laws of population growth. Proceedings of the National Academy of Sciences, National Acad Sciences, v. 105, n. 48, p. 18702-18707, 2008. Citado na página 39.

SONG, C.; KOREN, T.; WANG, P.; BARABÁSI, A.-L. Modeling the scaling properties of human mobility. arXiv preprint arXiv:1010.0436, 2010. Citado na página 39.

TOROCZKAI, Z.; GUCLU, H. Proximity networks and epidemics. Physica A: Statistical Mechanics and its Applications, Elsevier, v. 378, n. 1, p. 68-75, 2007. Citado na página 39.

VESPIGNANI, A. Predicting the behavior of techno-social systems. Science, American Association for the Advancement of Science, v. 325, n. 5939, p. 425-428, 2009. Citado na página 39.

YAMASAKI, K.; GOZOLCHIANI, A.; HAVLIN, S. Climate networks around the globe are significantly affected by el nino. Physical review letters, APS, v. 100, n. 22, p. 228501, 2008. Citado na página 23.

YANG, H.-X.; TANG, M.; WANG, Z. Suppressing epidemic spreading by risk-averse migration in dynamical networks. Physica A: Statistical Mechanics and its Applications, Elsevier, v. 490, p. 347-352, 2018. Citado na página 39. 


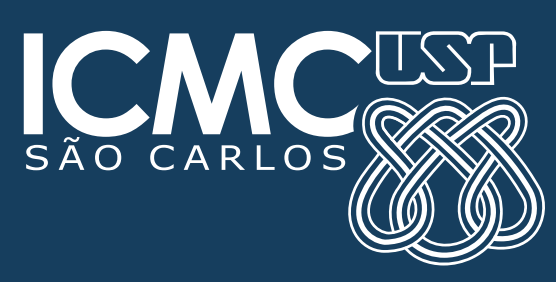

